\title{
SHADOWING ORBITS FOR TRANSITION CHAINS OF INVARIANT TORI ALTERNATING WITH BIRKHOFF ZONES OF INSTABILITY
}

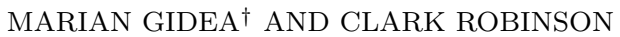

\begin{abstract}
We consider a dynamical system that exhibits transition chains of invariant tori alternating with Birkhoff zones of instability in a 2-dimensional center manifold. It is known that there exist orbits that shadow the transition chains. It is also known that there exist orbits that cross the Birkhoff zones of instability. We describe a topological mechanism that allows one to join together the two types of orbits. We prove the existence of diffusing orbits that shadow the transition chains of invariant tori and cross the Birkhoff zones of instability. This approach is motivated by what has been called the large gap problem in Hamiltonian systems.
\end{abstract}

\section{INTRODUCTION}

We present a topological mechanism for instability in normally hyperbolic dynamical systems. We consider a 2-dimensional normally hyperbolic manifold that contains a Cantor family of $C^{1}$-smooth invariant tori that can be grouped into transition chains alternating with Birkhoff zones of instability. Here by transition chains we mean finite sequences of tori such that each pair of successive tori have a transverse heteroclinic connection. On the Birkhoff zones of instability we assume no information whether or not there exist heteroclinic connection between their boundary components. It has been previously proved that, under suitable conditions, there exist orbits that shadow the transition chains; there also exist orbits that cross the Birkhoff zones of instability. In this paper we present a topological method that allows one to join together these two types of orbits. We show that there exist orbits starting near one torus from the Cantor family and ending near another torus, while they shadow the tori in each transition chain and alternatively cross the Birkhoff zones of instability separating consecutive transition chains.

1.1. Set-up and main result. Below we describe a dynamical system satisfying certain properties.

H0. We assume that $F: M \rightarrow M$ is a $C^{1}$-diffeomorphism of a smooth $d$ dimensional manifold $M$. We assume that there exists a compact invariant submanifold $A$ in $M$ that is diffeomorphic to an annulus $[0,1] \times \mathbb{T}^{1}$. We consider an action-angle coordinate system $(I, \phi)$ on $A$, with $I \in[0,1]$ and $\phi \in \mathbb{T}^{1}$. We assume that $A$ is normally hyperbolic, and for each point $p \in A$ the stable and unstable manifolds $W^{s}(p)$ and $W^{u}(p)$ are $n$-dimensional, with $2 n+2=d$. We also assume that the restriction $\left.F\right|_{A}$ of $F$ to $A$ is an area preserving monotone twist map.

1991 Mathematics Subject Classification. Primary, 37J40; 37C50; 37C29; Secondary, 37B30.

Key words and phrases. Arnold diffusion; correctly aligned windows; shadowing.

${ }^{\dagger}$ Research partially supported by NSF grant: DMS 0601016. 
We now assume the existence of certain objects that satisfy specific properties. The existence of those objects constitute the hypothesis of our main result, Theorem 1.2 , stated below. These numbered hypotheses will be followed by some comments, explanations, or definitions, which are not part of the hypotheses.

H1. Suppose that the annulus $A$ contains a Cantor family of $\left.F\right|_{A \text {-invariant, }}$ $C^{1}$-smooth, primary tori $\left\{T_{\iota}\right\}_{\iota \in \mathcal{I}}$, with the tori varying continuously in the $C^{1}$ topology.

By a primary torus (or, equivalently, an essential invariant circle) we mean a 1dimensional torus in $A$ that cannot be homotopically deformed into a point inside the annulus. See Figure 2.

To describe the relative positions of the tori $\left\{T_{\iota}\right\}_{\iota}$, we consider an order relation on $\mathcal{I}$, given by $\iota \prec \iota^{\prime}$ provided that the action coordinate $I$ of the intersection between the radius $\phi=$ const. and $T_{\iota}$ is less than the action coordinate $I^{\prime}$ of the intersection between the radius $\phi=$ const. and $T_{\iota^{\prime}}$. This order relation is independent of the choice of the radius $\phi=$ const., since invariant tori cannot intersect.

Since $F_{\mid A}$ is a monotone twist map, each torus $T_{\iota}$ is the graph of a Lipschitz function $\tau_{\iota}(\phi)$ (see Subsection 3.2); by H1, $\tau_{\iota}(\phi)$ is $C^{1}$-smooth. The fact that the tori are varying continuously in the $C^{1}$-topology means that $\tau_{\iota_{n}} \rightarrow \tau_{\iota}$ in the $C^{1}$ topology provided $\iota_{n} \rightarrow \iota$ as $n \rightarrow \infty$. Within the Cantor family $\left\{T_{\iota}\right\}_{\iota \in \mathcal{I}}$ one can distinguish two kinds of tori: 'interior' tori and 'boundary' tori. A torus $T_{\iota}$ is an 'interior' torus provided that there exist two sequences of tori $\left\{T_{\iota_{n}^{\prime}}\right\} \subseteq\left\{T_{\iota}\right\}$ with $\iota_{n}^{\prime} \prec \iota$, and $\left\{T_{\iota_{n}^{\prime \prime}}\right\} \subseteq\left\{T_{\iota}\right\}$ with $\iota \prec \iota_{n}^{\prime \prime}$, such that both sequences approach $T_{\iota}$ in the $C^{1}$-topology as $n \rightarrow \infty$. A torus $T_{\iota}$ is a 'boundary' torus provided that $T_{\iota}$ can be approximated only from above or only from below relative to the order $\prec$, meaning that there exists a sequence of tori $\left\{T_{\iota_{n}^{\prime}}\right\} \subseteq\left\{T_{\iota}\right\}$, with $\iota_{n}^{\prime} \prec \iota$, that approaches $T_{\iota}$ in the $C^{1}$-topology as $n \rightarrow \infty$, in which case there is no sequence of tori $\left\{T_{\iota_{n}^{\prime \prime}}\right\} \subseteq\left\{T_{\iota}\right\}$, with $\iota \prec \iota_{n}^{\prime \prime}$, that approaches $T_{\iota}$, or there exists a sequence of tori $\left\{T_{\iota_{n}^{\prime \prime}}\right\} \subseteq\left\{T_{\iota}\right\}$, with $\iota \prec \iota_{n}^{\prime \prime}$, that approaches $T_{\iota}$ in the $C^{1}$ topology as $n \rightarrow \infty$, in which case there is no sequence of tori $\left\{T_{\iota_{n}^{\prime}}\right\} \subseteq\left\{T_{\iota}\right\}$, with $\iota_{n}^{\prime} \prec \iota$, that approaches $T_{\iota}$.

For our main result, Theorem 1.2, we will choose and fix two tori $T_{\iota_{0}}$ and $T_{\iota_{1}}$ from $\left\{T_{\iota}\right\}_{\iota \in \mathcal{I}}$, with $\iota_{0} \prec \iota_{1}$.

H2. We assume that $\left.F\right|_{A}$ is topologically transitive on each torus from $\left\{T_{\iota}\right\}_{\iota \in \mathcal{I}}$.

From the normal hyperbolicity assumption, it follows that each invariant torus $T_{\iota}$ has $(n+1)$-dimensional stable and unstable manifolds $W^{s}\left(T_{\iota}\right)$ and $W^{u}\left(T_{\iota}\right)$.

When the unstable manifold $W^{u}\left(T_{\iota}\right)$ of a torus intersects transversally the stable manifold $W^{s}\left(T_{\iota}\right)$ of the same torus, we say that $T_{\iota}$ has a transverse homoclinic connection. When the unstable manifold $W^{u}\left(T_{\iota}\right)$ of a torus intersects transversally the stable manifold $W^{s}\left(T_{\iota^{\prime}}\right)$ of another torus $T_{\iota^{\prime}}$, we say that $T_{\iota}$ and $T_{\iota^{\prime}}$ have a transverse heteroclinic connection.

To state the next hypotheses, we will need to recall the notions of transition chain and of Birkhoff zone of instability.

We say that a finite sequence of tori $\left\{T_{\iota_{\kappa}}\right\}_{\kappa=0, \ldots, l} \subseteq\left\{T_{\iota}\right\}_{\iota}$ forms a (finite) transition chain if, for each $\kappa \in\{0, \ldots, l-1\}$, the unstable manifold $W^{u}\left(T_{\iota_{\kappa}}\right)$ of $T_{\iota_{\kappa}}$ intersects transversally the stable manifold $W^{s}\left(T_{\iota_{\kappa+1}}\right)$ of $T_{\iota_{\kappa+1}}$.

A region in $A$ between two tori $T_{\iota}$ and $T_{\iota^{\prime}}$ is called a Birkhoff zone of instability provided that there is no invariant primary torus in the interior of the region (see Definition 3.2). 


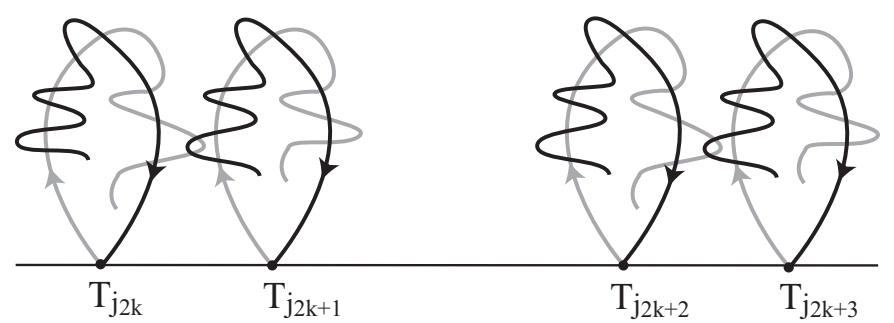

FIGURE 1. Transition chains of tori alternating with gaps.

For the Birkhoff zones of instability that we consider in this paper, the tori $T_{\iota}$ and $T_{\iota^{\prime}}$ at the boundary of a zone of instability are assumed to be from $\left\{T_{\iota}\right\}_{\iota}$; the region in $A$ between $T_{\iota}$ and $T_{\iota^{\prime}}$ contains no invariant primary torus, neither from $\left\{T_{\iota}\right\}_{\iota}$ nor in general.

H3. We assume that there exists an increasing sequence of indices in $\mathcal{I}$ given by $\iota_{0}=j_{0} \prec j_{1} \prec \ldots \prec j_{2 m} \prec j_{2 m+1}=\iota_{1}$ such that the following hold true:

H3.a. For each $k \in\{0, \ldots, m\}$ and for each pair $j^{\prime}, j^{\prime \prime} \in \mathcal{I}$ with $j_{2 k} \preceq j^{\prime} \prec j^{\prime \prime} \preceq$ $j_{2 k+1}$, there is a finite transition chain $\left\{T_{\iota_{\kappa}}\right\}_{\kappa=0, \ldots, l}$ with $j^{\prime}=\iota_{0} \prec \iota_{1} \prec$ $\ldots \prec \iota_{l-1} \prec \iota_{l}=j^{\prime \prime}$.

H3.b. The region in $A$ between $T_{j_{2 k+1}}$ and $T_{j_{2 k+2}}$ is a Birkhoff zone of instability, for $k \in\{0, \ldots, m-1\}$.

The hypothesis H3 says that the family of tori $\left\{T_{\iota}\right\}_{\iota \in \mathcal{I}}$ can be grouped into sequences of transition chains alternating with Birkhoff zones of instability. See Figure 1.2.

Remark 1.1. We emphasize that hypothesis $H 3$ does not prescribe whether or not there exist heteroclinic connections between the boundaries of the Birkhoff zones of instability specified in (H3.b). Therefore, in the future we will not assume that such heteroclinic connections exist. We will refer to these Birkhoff of instabilities as the 'large gaps'. We should also note there may exist other Birkhoff zones of instability besides those specified in (H3.b), between some consecutive pairs of tori that are part of the transition chains specified in (H3.a). However, the boundary tori of such Birkhoff zones of instability are assumed to have heteroclinic connections, as specified in (H3.a). Therefore, we will ignore this latter type of Birkhoff zones of instability in our future discussions.

The main result of this paper is the following:

Theorem 1.2. Assume that the system described in HO satisfies the conditions H1, H2, H3. Then, for each finite sequence of invariant tori $\left\{T_{i_{t}}\right\}_{t=0, \ldots, s}$ from $\left\{T_{\iota}\right\}_{\iota \in \mathcal{I}}$, with $T_{i_{0}}=T_{\iota_{0}}, T_{i_{s}}=T_{\iota_{1}}$, and for each finite sequence $\left\{\epsilon_{t}\right\}_{t=0, \ldots, s}$ of positive real numbers, there exist an orbit $\left\{z_{t}\right\}_{t=0, \ldots, s}$ and positive integers $\left\{n_{t}\right\}_{t=0, \ldots, s-1}$ such that

$$
\begin{gathered}
d\left(z_{t}, T_{i_{t}}\right)<\epsilon_{t}, \quad \text { for all } t=0, \ldots, s, \\
F^{n_{t}}\left(z_{t}\right)=z_{t+1}, \quad \text { for all } t=0, \ldots, s-1 .
\end{gathered}
$$

The proof of this theorem is based on the topological method of correctly aligned windows. The main idea is to construct a sequence of windows (homeomorphic 
copies of multi-dimensional rectangles) that 'crawl across' the Birkhoff zones instability and 'jump along' the transition chains. Then a 'shadowing lemma' type of argument implies the existence of true orbits that shadow the given sequence of windows.

Remark 1.3. The verification of the hypothesis of Theorem 1.2 in concrete examples can be quite challenging. The existence of transverse heteroclinic connections is usually verified through a Melnikov method [10]. This yields the existence of transition chains of invariant tori. Some methods to verify the existence of Birkhoff zones of instability are discussed in [2, 22, 25, 31, 32].

However, we would like to think of Theorem 1.2 essentially as a simplified model for the existence of diffusing orbits.

Since the method of correctly aligned windows that we engage here is purely topological, we hope that we will be able to adapt it to the study of Arnold diffusion under typical assumption. One of the main difficulties in applying Theorem 1.2 to concrete systems is that the boundaries of the Birkhoff zones of instability are in general only Lipschitz. These Lipschitz tori are in general limits of $C^{1}$-smooth tori. It would be possible to construct finite sequences of correctly aligned windows along the transition chains such that the windows that reach these limiting $C^{1}$ tori, at the end of the transition chains, cross inside the Birkhoff zone of instability; then, these sequences of correctly aligned windows will be joined together with windows that cross the Birkhoff zone of instability. This strategy will be explored in a future research.

Example 1.4. We outline an argument that provides examples of dynamical systems that satisfy the hypotheses of Theorem 1.2. We first describe how is it possible to construct a twist map of the annulus for which the Birkhoff zones of instability have $C^{1}$-smooth boundaries. Then we take the product of such a twist map with a time-discretization of a pendulum and apply a small perturbation.

Sylvain Crovisier sent us a personal communication ([9]) on how to use his results with Christian Bonatti ([5]) to construct an example of a twist map with one Birkhoff zone of instability with $C^{1}$-smooth boundary components. Their version of the connecting lemma (following Hayashi) constructs a $C^{1}$ symplectic diffeomorphism with a dense orbit on a compact manifold without boundary. The proof involves the construction of a finite collection of small perturbation boxes and of a $C^{1}$ perturbation with the support inside the boxes. However, we want to apply their method to an open annulus. We need to use an expanding sequence of closed annuli whose union gives the open annulus between two invariant circles. There is a pseudo-orbit ( $\epsilon$-chain) that is dense in each of these annuli. Applying their perturbation boxes technique, there is a $C^{1}$ symplectic diffeomorphism with a $(1 / n)$-dense actual orbit in the $n$-th annulus. Taking the limit gives a $C^{1}$ symplectic diffeomorphism that has the boundaries still the same, so smooth, and an actually dense orbit in the open annulus.

To construct a $C^{1}$ symplectic diffeomorphism with a Cantor set of invariant circles, we need to use the above result on a sequence of open annuli. Start with a linear twist map on an annulus. We look at the parameter space $\mathcal{I}$ for the invariant tori (circles). There is a countable set of parameters that correspond to rational rotations. Put an open interval $J_{k}$ about each of parameters in $\mathcal{I}$ such that $\mathcal{I} \backslash \bigcup J_{k}$ is a Cantor set. (This is a matter of choosing the lengths in a way that does not leave any isolated points.) Now on the sequence of annuli that correspond to 


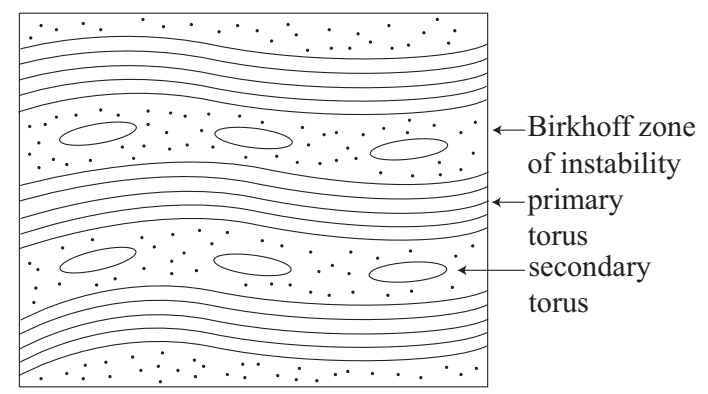

Figure 2. Primary tori, secondary tori, and Birkhoff zones of instability.

the sequence of $J_{k}$, construct a perturbation of the type indicated in the previous paragraph. This can be done to make a $C^{1}$ symplectic diffeomorphism that leave the map on the tori corresponding to $\mathcal{I} \backslash \bigcup J_{k}$ the same as before, and has a dense orbit in each of the open annuli that corresponds to one of the $J_{k}$. This constructs an approximation that has a Cantor set of smooth invariant tori with irrational rotations and each $J_{k}$ corresponding to a Birkhoff zone of instability. This ends the sketch of the construction of an example of a twist map that has Birkhoff zones of instability with $C^{1}$-smooth boundaries.

To construct an example of a system that satisfies the hypothesis of Theorem 1.2, we can take the product of the twist map described above with a time-discretization of a pendulum system. The stable and unstable manifolds of the hyperbolic fixed point of the pendulum coincide. We can apply a perturbation that does not affect the annulus and such that the stable and unstable manifolds have a transverse homoclinic connection. This implies that all invariant tori that are within a certain distance (depending on the size of the splitting of the stable and unstable manifold) have a transverse heteroclinic connection. In this way, the Cantor family of tori can be organized into transition chains of tori alternating with Birkhoff zones of instability.

1.2. Motivation. The motivation for this study comes from the large gap problem of Arnold diffusion in Hamiltonian systems. One formulation of this problem considers perturbations of a priori unstable Hamiltonian systems, of the form

$$
H_{\mu}(I, \phi, s, u)=H_{0}(I)+H_{1}(s, u)+\mu H_{2}(I, \phi, s, u ; \mu),
$$

where $(I, \phi) \in \mathbb{R}^{m+1} \times \mathbb{T}^{m+1}$ and $(s, u) \in \mathbb{R}^{n} \times \mathbb{R}^{n}$; for such systems, one is interested in the existence of orbits that travel for a distance of order $O(1)$ in the action variable $I$, for each $\mu$ with $0<\mu<\mu_{*}$, where $\mu_{*}>0$ is sufficiently small and fixed.

The unperturbed system

$$
H_{0}(I)+H_{1}(s, u)
$$

is described in terms of action-angle variables $(I, \phi)$ and hyperbolic variables $(s, u)$. It is usually assumed that the Hamiltonian system

$$
\begin{aligned}
& \frac{d u}{d t}=\frac{\partial H_{1}}{\partial s} \\
& \frac{d s}{d t}=-\frac{\partial H_{1}}{\partial u}
\end{aligned}
$$


has a hyperbolic fixed point that is connected to itself by a homoclinic orbit. By restricting to an energy level and taking an appropriate Poincaré section $M_{\mu}$ for each $\mu$, one obtains a diffeomorphism $F_{\mu}$ acting on a $(2 n+2 m)$-dimensional manifold $M_{\mu}$. This manifold $M_{\mu}$ contains a $(2 m)$-dimensional normally hyperbolic manifold, foliated by an $m$-parameter family of $m$-dimensional tori, each having $(m+n)$ dimensional stable and unstable manifolds. It is usually assumed that $H_{0}$ satisfies an isoenergetic non-degeneracy condition, that

$$
\left(\begin{array}{cc}
\partial^{2} H_{0} / \partial I^{2} & \partial H_{0} / \partial I \\
\partial H_{0} / \partial I & 0
\end{array}\right)
$$

is non-singular, which implies that the map $F_{0}$, corresponding to the unperturbed system, satisfies a twist condition in the $(I, \phi)$ variables. Then, by the KAM theorem, there exists a Cantor set $\mathcal{I}_{\mu}$ of smooth $m$-dimensional tori that survive the perturbation for each $0<\mu<\mu_{1}$, for some $\mu_{1}>0$. Under certain conditions, the KAM tori vary continuously in the $C^{1}$-topology (see [36]). The motion along the KAM tori is quasi-periodic. Besides the KAM tori, there exist in general some other invariant primary tori, not necessarily smooth, that survive the perturbation. (See [23]. Some interesting results on non-smooth tori at the boundary of renormalization can be found in [28, 39].) The surviving invariant tori are separated by gaps. The typical size of the large gaps is of order $O\left(\mu^{1 / 2}\right)$.

It is also usually assumed that the perturbation $\mathrm{H}_{2}$ satisfies a Melnikov-type of condition. Such a condition ensures that for each $0<\mu<\mu_{2}$, for some $0<\mu_{2}<\mu_{1}$, there exists $\delta_{\mu}>0$, depending on the value of the perturbation parameter $\mu$, such that any two tori within a distance of $\delta_{\mu}$ are linked by a transverse heteroclinic connection. When $\mu \rightarrow 0$, we have $\delta_{\mu} \rightarrow 0$. Typically, the splitting size of the stable and unstable manifolds is of order $O(\mu)$. Since the size of the gaps is of order $O\left(\mu^{1 / 2}\right)$, one cannot form transition chains of primary invariant tori that cross those gaps.

Suppose that we choose two distinct tori $T_{\iota_{0}}$ and $T_{\iota_{1}}$, depending on $\mu$, that survive the perturbation and that lie at a distance of order $O(1)$ in the action direction. For any fixed, sufficiently small value of $\mu$, the invariant tori surviving the perturbation can be ordered in groups of tori linked by transverse heteroclinic connections, alternating with large gaps, which contain no primary invariant tori. The number of the large gaps is finite for any fixed perturbation size $\mu$. The large gap problem of Arnold diffusion is to show that there exists $0<\mu_{*}<\mu_{2}$ such that for each $0<\mu<\mu_{*}$ there exists an orbit that, despite of the large gaps, travels from near $T_{\iota_{0}}$ to near $T_{\iota_{1}}$.

In this paper we consider a simplified model for Arnold diffusion. Our model assumes that some of the geometric objects that occur in perturbed Hamiltonians are already given: there exists a normally hyperbolic manifold which contains a Cantor family of $C^{1}$-smooth invariant tori, and there exist transverse heteroclinic connections between pairs of sufficiently close tori (hypothesis H3.a). The large gaps are modelled by the Birkhoff zones of instability for which there is no information on the existence of heteroclinic connections between the boundary components (hypothesis H3.b). The simplifying assumptions are that the normally invariant manifold is 2-dimensional (rather than $(2 m)$-dimensional), and the tori at the boundary of the Birkhoff zones of instability modelling the large gaps are $C^{1}$-smooth. The fact that the normally invariant manifold is assumed to be a 2-dimensional annulus allows us to make use of the existence of (Birkhoff) orbits that travel from an arbitrarily 
small neighborhood of one boundary of a Birkhoff zone of instability to an arbitrarily small neighborhood of the other boundary. In higher dimension, applying similar ideas is more difficult (see, for example, [21]). The requirement that the boundaries of the Birkhoff zones of instability are $C^{1}$-smooth is drastic (in general, they are only known to be Lipschitz). However, the condition is mainly used to simplify the construction of windows, and to avoid more technical assumptions on the transition chains. Therefore, we hope that this restriction can be removed from our argument.

The general scheme of the proof of Theorem 1.2 is the following. We are given a finite sequence of tori that we want to shadow. We augment this sequence with other tori from the Cantor family, and with all the tori at the boundaries of the Birkhoff zones of instability that model the large gaps. It is now the augmented sequence of tori that we want to shadow. We construct correctly aligned windows along the transition chains of tori from the augmented sequence and we also construct correctly aligned windows that cross the Birkhoff zones of instability (following Birkhoff orbits). When these two types of correctly aligned windows meet at the boundaries of the Birkhoff zones of instability, we make them intersect in a manner that is correctly aligned. The fact that these boundaries are assumed to be $C^{1}$ smooth simplifies considerably this construction. In the process of constructing correctly aligned windows, an important feature of the Cantor family of tori that we use is that the tori can be approximated by other tori from the family: thus, the windows constructed between pairs of nearby tori can be controlled for a long time under the twist dynamics. Once a finite sequence of correctly aligned tori is constructed from the initial torus to the final torus, a shadowing lemma type of result provides us with an orbit which visits all the constructed windows, and thus shadows the augmented sequence of tori.

Remark 1.5. Recent progress in the large gap problem has been achieved through geometric methods by Delshams, de la Llave, and Seara [12], and through variational methods by Cheng and Yan [6, 7]. See also the geometric mechanism for Arnold diffusion developed by Treschev in [40, 41], and the announcements of other variational methods by Z. Xia [44] and by J. Mather [34]. A topological argument for the large gap problem, relying on some of the results in [12], was provided in [19]. An independent topological argument for the large gap problem, which also yields an optimal estimate of the diffusion time, has been carried out in [20]. Treatments related to ours can also be found in $[26,35]$.

\section{Correctly aligned windows}

We give a brief exposition of a topological version of Easton's method of correctly aligned windows [14], following the ideas from [19, 42, 43]. We will omit most of the proofs, which can be found in the above mentioned references.

Let $f: M \rightarrow M$ be a continuous mapping on a smooth $n$-dimensional manifold $M$.

Definition 2.1. A window $W$ is a homeomorphic copy $c_{W}\left([0,1]^{u} \times[0,1]^{s}\right)$ in $M$ of a multi-dimensional rectangle $[0,1]^{u} \times[0,1]^{s}$ with specified expanding- and contracting-like directions of dimensions $u, s>0$ respectively, where $u+s=n$, together with the underlying homeomorphism $c_{W}: \operatorname{dom}\left(c_{W}\right) \rightarrow \operatorname{im}\left(c_{W}\right)$, where $\operatorname{dom}\left(c_{W}\right)$ is an open neighborhood of $[0,1]^{u} \times[0,1]^{s} \subseteq \mathbb{R}^{n}$, and $\operatorname{im}\left(c_{W}\right)$ is an open 
neighborhood of $W$ in $M$. The boundary components $W^{-}=c_{W}\left(\partial[0,1]^{u} \times[0,1]^{s}\right)$ and $W^{+}=c_{W}\left([0,1]^{u} \times \partial[0,1]^{s}\right)$ will be referred as the exit and the entry set, respectively.

We will often refer to the image $c_{W}\left([0,1]^{u} \times[0,1]^{s}\right)$ of the rectangle $[0,1]^{u} \times$ $[0,1]^{s}$ through $c_{W}$ as to the window $W$ itself. The components of the mapping $c_{W}^{-1}: \operatorname{im}\left(c_{W}\right) \rightarrow \operatorname{dom}\left(c_{W}\right)$ will be referred to as the local coordinates of $W$. We also denote $(W)_{c_{W}}=c_{W}^{-1}(W)$. When we want to specify the dimensions $u, s$ of the expanding- and contracting-like dimensions, we will refer to $W$ as a $(u, s)$ window. We will refer to the directions tangent to the images through $c$ of the sheets $[0,1]^{u} \times\{y\}$, where $y \in[0,1]^{s}$, as the exit directions. We will also refer to the entry directions of a window, which have a similar description.

Definition 2.2. Let $W_{1}$ and $W_{2}$ be two windows in $M$ for which the corresponding expanding- and contracting-like directions have the same dimensions, and such that $f\left(W_{1}\right) \subseteq \operatorname{im}\left(c_{W_{2}}\right)$. Let $f_{c}: \operatorname{dom}\left(c_{W_{1}}\right) \rightarrow \operatorname{dom}\left(c_{W_{2}}\right)$ be given by $f_{c}=c_{W_{2}}^{-1} \circ f \circ c_{W_{1}}$. By definition, the window $W_{1}$ is correctly aligned with the window $W_{2}$ under $f$ provided that there exist $0 \leq a_{i}<b_{i} \leq 1, i=1, \ldots, u$, such that:

(i) There exists a sub-rectangle of $\left(W_{1}\right)_{c_{W_{1}}}$ which is mapped by $f_{c}$ away from the entry set of $\left(W_{2}\right)_{c_{W_{2}}}$, and whose exit set is mapped by $f_{c}$ away from the whole of $\left(W_{2}\right)_{c_{W_{2}}}$, i.e.,

$$
\begin{aligned}
& f_{c}\left(\left(\Pi_{i=1}^{u}\left[a_{i}, b_{i}\right]\right) \times[0,1]^{s}\right) \cap\left([0,1]^{u} \times \partial[0,1]^{s}\right)=\emptyset \\
& f_{c}\left(\partial\left(\Pi_{i=1}^{u}\left[a_{i}, b_{i}\right]\right) \times[0,1]^{s}\right) \cap\left([0,1]^{u} \times[0,1]^{s}\right)=\emptyset .
\end{aligned}
$$

(ii) The image under $f_{c}$ of the sub-rectangle of $\left(W_{1}\right)_{c_{W_{1}}}$ from (i) crosses $\left(W_{2}\right)_{c_{W_{2}}}$ in a topologically non-trivial way, in the sense that there exists a continuous homotopy $h_{t}: \operatorname{dom}\left(c_{W_{1}}\right) \rightarrow \operatorname{dom}\left(c_{W_{2}}\right)$, with $t \in[0,1]$, such that the following conditions hold true:

$$
\begin{aligned}
h_{0} & =f_{c}, \\
h_{[0,1]}\left(\left(\Pi_{i=1}^{u}\left[a_{i}, b_{i}\right]\right) \times[0,1]^{s}\right) \cap\left([0,1]^{u} \times \partial[0,1]^{s}\right) & =\emptyset, \\
h_{[0,1]}\left(\partial\left(\Pi_{i=1}^{u}\left[a_{i}, b_{i}\right]\right) \times[0,1]^{s}\right) \cap\left([0,1]^{u} \times[0,1]^{s}\right) & =\emptyset,
\end{aligned}
$$

and there exists a linear map $A: \mathbb{R}^{u} \rightarrow \mathbb{R}^{u}$, such that

- $h_{1}(x, y)=(A x, 0)$ for all $x \in[0,1]^{u}$ and $y \in[0,1]^{s}$,

- $A\left(\partial[0,1]^{u}\right) \subset \mathbb{R}^{u} \backslash[0,1]^{u}$,

- $\operatorname{det}(A) \neq 0$.

See Figure 3 (for $u=s=1$ ).

Remark 2.3. In the above definition, the fact that a sub-rectangle of $\left(W_{1}\right)_{c_{W_{1}}}$ crosses $\left(W_{2}\right)_{c_{W_{2}}}$ in a non-trivial way means that one can homotopically deform the image of the sub-rectangle into a $u$-dimensional plane given by a non-trivial linear map that depends only on the expanding-like variables. More generally, one can require that the sub-rectangle can be transformed, through a homotopy deformation, into a $u$-dimensional surface described by a mapping of non-zero Brouwer degree. We will not pursue this alternative here; see [43] for details.

Also, we remark that in [43] a sightly less general situation is considered, namely when the whole window $W_{1}$ is stretched across $W_{2}$ in a non-trivial way - this corresponds to the case in Definition 2.2 when $a_{i}=0$ and $b_{i}=1$ for all $i=1, \ldots, u$. However, as we will see below, the more general case discussed here can be easily 


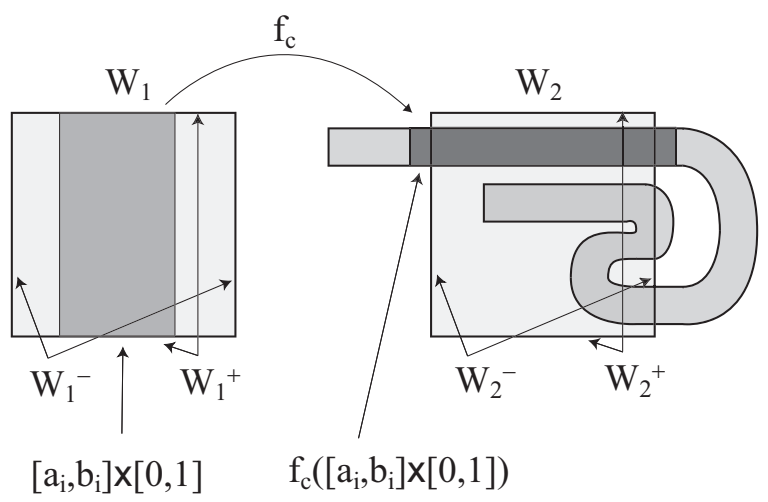

FIGURE 3. Correctly aligned windows.

reduced to the special case discussed in [43]. To distinguish between the more general case described in Definition 2.2 from the special case considered in [43], we will use the term 'correct alignment' to describe the situation in Definition 2.2, and the term 'covering relation' to describe the situation in [43].

For 2-dimensional windows, we have the following easy criterion to check for correct alignment.

Proposition 2.4. Assume that $W_{1}$ and $W_{2}$ are 2-dimensional windows on a surface, and $u=s=1$. Then $W_{1}$ is correctly aligned with $W_{2}$ under $f$ if $f\left(W_{1}\right) \subseteq$ $i m\left(c_{W_{2}}\right)$ and there exist $0 \leq a<b \leq 1$ such that the following hold true:

(i) $f_{c}([a, b] \times[0,1]) \subseteq \mathbb{R} \times(0,1)$.

(ii) Either $f_{c}^{1}(\{a\} \times[0,1])<0$ and $f_{c}^{1}(\{b\} \times[0,1])>1$, or $f_{c}^{1}(\{a\} \times[0,1])>1$ and $f_{c}^{1}(\{b\} \times[0,1])<0$, where $f_{c}=\left(f_{c}^{1}, f_{c}^{2}\right)$.

The situation described by this proposition is depicted in Figure 3.

The next result is a topological version of the shadowing lemma for hyperbolic systems.

Theorem 2.5. Let $f_{i}: M \rightarrow M$ be a family of continuous maps and $W_{i}$ be a family of windows for which the corresponding expanding- and contracting-like directions have the same dimensions, where $i \in \mathbb{Z}$. Assume that, for each $i$, we have $W_{i}$ correctly aligned with $W_{i+1}$ under $f_{i}$. Then there exists a point $x \in W_{0}$, such that $\left(f_{i} \circ f_{i-1} \circ \cdots \circ f_{1}\right)(x) \in W_{i}$ for all $i \in \mathbb{Z}$.

Proof. The proof reduces to the proof of Theorem 4 in [43], once we show that the more general case of correct alignment described by Definition 2.2 reduces to the special case considered in [43]. The special case means that the sub-rectangle specified in Definition 2.2 (i) is in fact the whole rectangle, that is, $a_{i}=0$ and $b_{i}=1$, for all $i=1, \ldots, u$.

Assume that $W_{i}$ is correctly aligned with $W_{i+1}$ under $f_{i}$. Then, there exists a sub-window $W_{i}^{\natural} \subseteq W_{i}$, corresponding to a rectangle of the form $\left(\Pi_{j=1}^{u}\left[a_{j}^{\natural}, b_{j}^{\natural}\right]\right) \times$ $[0,1]^{s}$, such that $W_{i}^{\natural}$ satisfies a covering relation with $W_{i+1}$ under $f_{i}$. By slightly deforming the original $W_{i}$ we can ensure that $W_{i}$ satisfies a covering relation with $W_{i}^{\natural}$ under the identity mapping, while all the correct alignment conditions on the 
windows preceding $W_{i}$ and on the windows succeeding $W_{i+1}$ in the sequence remain unaltered. Thus, we replaced the correct alignment of $W_{i}$ with $W_{i+1}$ with a sequence of two covering relations, from $W_{i}$ to $W_{i}^{\natural}$, and from $W_{i}^{\natural}$ to $W_{i+1}$.

In the rest of this section we outline a convenient method to verify correct alignment of windows in higher dimensions. Given two windows and a map, if each window can be written as a product of window components, and if the components of the first window are correctly aligned with the corresponding components of the second window under the appropriate components of the map, then the first window is correctly aligned with the second window under the given map.

Suppose that $u_{1}, u_{2}, s_{1}, s_{2}>0$ and $u_{1}+u_{2}+s_{1}+s_{2}=n$. Let $u_{1}+u_{2}=u$, $s_{1}+s_{2}=s, u_{1}+s_{1}=n_{1}$, and $u_{2}+s_{2}=n_{2}$. We describe points in $\mathbb{R}^{n}$ by their coordinates $\left(x_{1}, x_{2}, y_{1}, y_{2}\right)$ where $x_{1} \in \mathbb{R}^{u_{1}}, x_{2} \in \mathbb{R}^{u_{2}}, y_{1} \in \mathbb{R}^{s_{1}}$, and $y_{2} \in \mathbb{R}^{s_{2}}$. We denote by $\pi_{\left(x_{1}, y_{1}\right)}$ the projection mapping onto the $\left(x_{1}, y_{1}\right)$-coordinates, and by $\pi_{\left(x_{2}, y_{2}\right)}$ the projection mapping onto the $\left(x_{2}, y_{2}\right)$-coordinates.

Let $W_{1}$ be a window in $M$ of expanding-like direction of dimension $u$ and contracting-like direction of dimension $s$, and let $c_{W_{1}}$ be the underlying homeomorphism. For each $\left(x_{2}^{0}, y_{2}^{0}\right) \in[0,1]^{u_{2}} \times[0,1]^{s_{2}}$, the mapping $c_{W_{1}}^{\left(x_{2}^{0}, y_{2}^{0}\right)}$ defined by

$$
\left(x_{1}, y_{1}\right) \in[0,1]^{u_{1}} \times[0,1]^{s_{1}} \rightarrow c_{W_{1}}^{\left(x_{2}^{0}, y_{2}^{0}\right)}\left(x_{1}, y_{1}\right):=c_{W_{1}}\left(x_{1}, x_{2}^{0}, y_{1}, y_{2}^{0}\right)
$$

is a homeomorphism, defining a $\left(u_{1}, s_{1}\right)$-window which we denote $W_{1}^{\left(x_{2}^{0}, y_{2}^{0}\right)}$. The exit set of this window is defined as

$$
\left(W_{1}^{\left(x_{2}^{0}, y_{2}^{0}\right)}\right)^{-}=c_{W_{1}}^{\left(x_{2}^{0}, y_{2}^{0}\right)}\left(\partial[0,1]^{u_{1}} \times[0,1]^{s_{1}}\right) .
$$

Its entry set is defined similarly.

Also, for each $\left(x_{1}^{0}, y_{1}^{0}\right) \in[0,1]^{u_{1}} \times[0,1]^{s_{1}}$, the mapping $c_{W_{1}}^{\left(x_{1}^{0}, y_{1}^{0}\right)}$ defined by

$$
\left(x_{2}, y_{2}\right) \in[0,1]^{u_{2}} \times[0,1]^{s_{2}} \rightarrow c_{W_{1}}^{\left(x_{1}^{0}, y_{1}^{0}\right)}\left(x_{2}, y_{2}\right):=c_{W_{1}}\left(x_{1}^{0}, x_{2}, y_{1}^{0}, y_{2}\right)
$$

is a homeomorphism, defining a $\left(u_{2}, s_{2}\right)$-window which we denote $W_{1}^{\left(x_{1}^{0}, x_{2}^{0}\right)}$. The exit set of this window is defined as

$$
\left(W_{1}^{\left(x_{1}^{0}, y_{1}^{0}\right)}\right)^{-}=c_{W_{1}}^{\left(x_{1}^{0}, y_{1}^{0}\right)}\left(\partial[0,1]^{u_{2}} \times[0,1]^{s_{2}}\right) .
$$

Its entry set is defined similarly.

When we write these windows in local coordinates, we have

$$
\begin{aligned}
&\left(W_{1}^{\left(x_{2}^{0}, y_{2}^{0}\right)}\right)_{c}:=\left(c_{W_{1}}^{\left(x_{2}^{0}, y_{2}^{0}\right)}\right)^{-1}\left(W_{1}^{\left(x_{2}^{0}, y_{2}^{0}\right)}\right)=[0,1]^{u_{1}} \times[0,1]^{s_{1}}, \\
&\left(\left(W_{1}^{\left(x_{2}^{0}, y_{2}^{0}\right)}\right)^{-}\right)_{c}:=\left(c_{W_{1}}^{\left(x_{2}^{0}, y_{2}^{0}\right)}\right)^{-1}\left(\left(W_{1}^{\left(x_{2}^{0}, y_{2}^{0}\right)}\right)^{-}\right)=\partial[0,1]^{u_{1}} \times[0,1]^{s_{1}}, \\
&\left(W_{1}^{\left(x_{1}^{0}, y_{1}^{0}\right)}\right)_{c}:=\left(c_{W_{1}}^{\left(x_{1}^{0}, y_{1}^{0}\right)}\right)^{-1}\left(W_{1}^{\left(x_{1}^{0}, y_{1}^{0}\right)}\right)=[0,1]^{u_{2}} \times[0,1]^{s_{2}}, \\
&\left(\left(W_{1}^{\left(x_{1}^{0}, y_{1}^{0}\right)}\right)^{-}\right)_{c}:=\left(c_{W_{1}}^{\left(x_{1}^{0}, y_{1}^{0}\right)}\right)^{-1}\left(\left(W_{1}^{\left(x_{1}^{0}, y_{1}^{0}\right)}\right)^{-}\right)=\partial[0,1]^{u_{2}} \times[0,1]^{s_{2}} .
\end{aligned}
$$

In the above notation, the subscript $c$ stands for the appropriate homeomorphism associated to the window under consideration. 


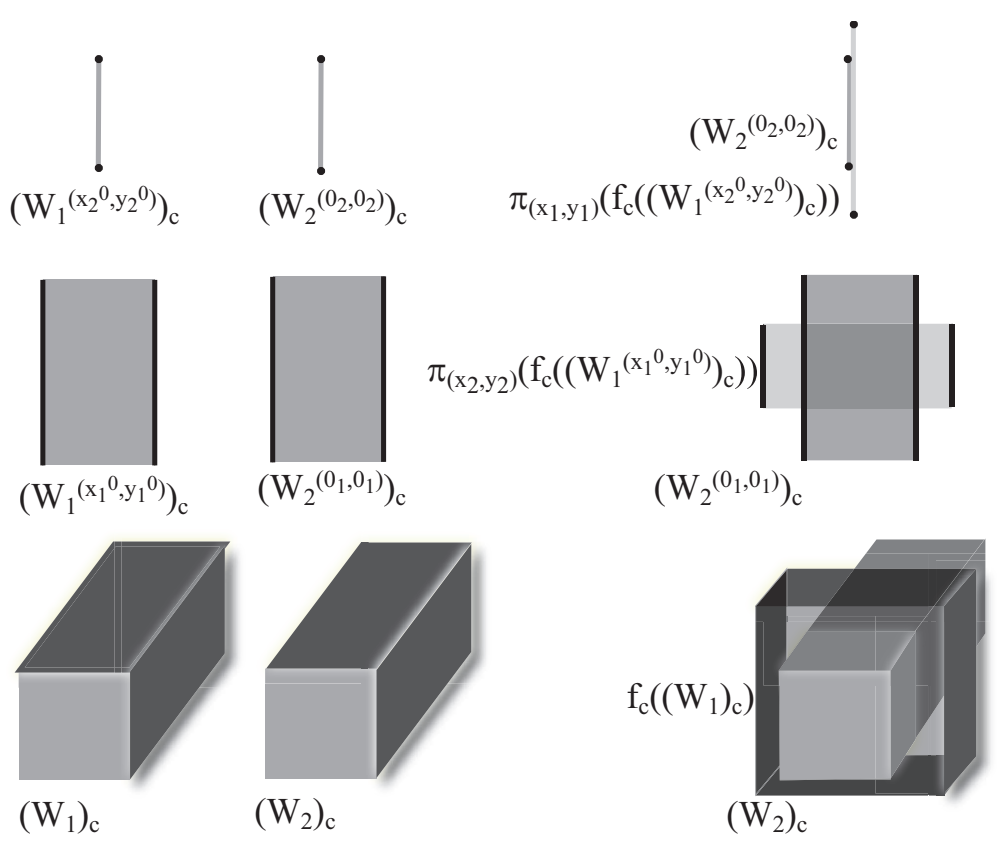

FiguRE 4. A product of correctly aligned windows. The exit set of each window is shown in darker color.

Thus, we can write $W_{1}$ in local coordinates as

$$
\begin{aligned}
\left(W_{1}\right)_{c}= & \left(W_{1}^{\left(x_{2}^{0}, y_{2}^{0}\right)}\right)_{c} \times\left(W_{1}^{\left(x_{1}^{0}, y_{1}^{0}\right)}\right)_{c}, \\
\left(W_{1}^{-}\right)_{c}= & {\left[\left(\left(W_{1}^{\left(x_{2}^{0}, y_{2}^{0}\right)}\right)^{-}\right)_{c} \times\left(W_{1}^{\left(x_{1}^{0}, y_{1}^{0}\right)}\right)_{c}\right] } \\
& \cup\left[\left(W_{1}^{\left(x_{2}^{0}, y_{2}^{0}\right)}\right)_{c} \times\left(\left(W_{1}^{\left(x_{1}^{0}, y_{1}^{0}\right)}\right)^{-}\right)_{c}\right] .
\end{aligned}
$$

for any $\left(x_{2}^{0}, y_{2}^{0}\right) \in[0,1]^{u_{2}} \times[0,1]^{s_{2}}$ and $\left(x_{1}^{0}, y_{1}^{0}\right) \in[0,1]^{u_{1}} \times[0,1]^{s_{1}}$. The entry set of $W_{1}$ can be described in a similar fashion.

Thus, in local coordinates, the window $W_{1}$ is the product of its components $W_{1}^{\left(x_{2}^{0}, y_{2}^{0}\right)}$ and $W_{1}^{\left(x_{1}^{0}, y_{1}^{0}\right)}$. Note that the exit set of $W_{1}$ is not, in local coordinates, the product of the exit sets of $W_{1}^{\left(x_{2}^{0}, y_{2}^{0}\right)}$ and $W_{1}^{\left(x_{1}^{0}, y_{1}^{0}\right)}$.

Let us now consider a second window $W_{2}$ in $M$, for which the expanding- and contracting-like directions have the same dimensions as those corresponding to $W_{1}$, and let $c_{W_{2}}$ be the underlying homeomorphism. The window $W_{2}$ is, in local coordinates, the product of the component windows $W_{2}^{\left(0_{2}, 0_{2}\right)}$, corresponding to $\left(x_{2}^{0}, y_{2}^{0}\right)=(0,0)$, and $W_{2}^{\left(0_{1}, 0_{1}\right)}$, corresponding to $\left(x_{1}^{0}, y_{1}^{0}\right)=(0,0)$.

We now describe a situation in which the correct alignment of the windows $W_{1}^{\left(x_{2}^{0}, y_{2}^{0}\right)}$ and $W_{2}^{\left(0_{2}, 0_{2}\right)}$, and of the windows $W_{1}^{\left(x_{1}^{0}, y_{1}^{0}\right)}$ and $W_{2}^{\left(0_{1}, 0_{1}\right)}$, implies the correct alignment of the windows $W_{1}$ and $W_{2}$. 
Proposition 2.6. Let $W_{1}$ and $W_{2}$ be $(u, s)$-windows as above. Assume:

(i) For each $\left(x_{2}^{0}, y_{2}^{0}\right) \in[0,1]^{u_{2}} \times[0,1]^{s_{2}}$, the $\left(u_{1}, s_{1}\right)$-window $\left(W_{1}^{\left(x_{2}^{0}, y_{2}^{0}\right)}\right)_{c}$ is correctly aligned with the $\left(u_{1}, s_{1}\right)$-window $\left(W_{2}^{\left(0_{2}, 0_{2}\right)}\right)_{c}$ under $\pi_{\left(x_{1}, y_{1}\right)} \circ f_{c}$.

(ii) For each $\left(x_{1}^{0}, y_{1}^{0}\right) \in[0,1]^{u_{1}} \times[0,1]^{s_{1}}$, the $\left(u_{2}, s_{2}\right)$-window $\left(W_{1}^{\left(x_{1}^{0}, y_{1}^{0}\right)}\right)_{c}$ is correctly aligned with the $\left(u_{2}, s_{2}\right)$-window $\left(W_{2}^{\left(0_{1}, 0_{1}\right)}\right)_{c}$ under $\pi_{\left(x_{2}, y_{2}\right)} \circ f_{c}$.

Then $W_{1}$ is correctly aligned with $W_{2}$ under $f$.

See Figure 4.

Remark 2.7. The fact that, in the above proposition, we fixed the coordinates $\left(x_{2}^{0}, y_{2}^{0}\right)$ and $\left(x_{1}^{0}, y_{1}^{0}\right)$ to $(0,0)$ in the components of the window $W_{2}$ is a matter of convenience; one can describe more complicated condition under which the correct alignment of windows can occur. However, the above conditions can be easier to verify in certain situations. For example, if one knows that $\left(W_{1}^{\left(0_{2}, 0_{2}\right)}\right)_{c}$ is correctly aligned with $\left(W_{2}^{\left(0_{2}, 0_{2}\right)}\right)_{c}$, and $\left(W_{1}^{\left(0_{1}, 0_{1}\right)}\right)_{c}$ is correctly aligned with $\left(W_{2}^{\left(0_{1}, 0_{1}\right)}\right)_{c}$, one can argue that, by continuity, $\left(W_{1}^{\left(x_{2}^{0}, y_{2}^{0}\right)}\right)_{c}$ is correctly aligned with $\left(W_{2}^{\left(0_{2}, 0_{2}\right)}\right)_{c}$ for all $\left(x_{2}^{0}, y_{2}^{0}\right)$ sufficiently close to $(0,0)$, and $\left(W_{1}^{\left(x_{1}^{0}, y_{1}^{0}\right)}\right)_{c}$ is correctly aligned with $\left(W_{2}^{\left(0_{1}, 0_{1}\right)}\right)_{c}$ for all $\left(x_{1}^{0}, y_{1}^{0}\right)$ sufficiently close to $(0,0)$. If the window $W_{1}$ can be made sufficiently small in size (at least in some directions), this continuity argument is sufficient to conclude that $W_{1}$ is correctly aligned with $W_{2}$.

\section{Preliminaries to the Proof}

3.1. Normal hyperbolicity. The assumption made in Section 4.2 that $A$ in normally hyperbolic in $M$ relative to $F$ means that $T M$ can be split into $(D F)$-invariant sub-bundles

$$
T M=T A \oplus E^{s} \oplus E^{u},
$$

and there exist a Riemannian metric on $M$, a constant $C>0$, and rates $0<\lambda<$ $\mu^{-1}<1$ such that for all $x \in A$ and $v \in T_{p} M$ we have

$$
\begin{gathered}
v \in E_{x}^{s} \Rightarrow\left\|\left(D F^{n}\right)_{p}(v)\right\| \leq C \lambda^{n}\|v\|, \text { for all } n \geq 0, \\
v \in E_{x}^{u} \Rightarrow\left\|\left(D F^{n}\right)_{p}(v)\right\| \leq C \lambda^{-n}\|v\|, \text { for all } n \leq 0, \\
v \in T A \Rightarrow\left\|\left(D F^{n}\right)_{p}(v)\right\| \leq C \mu^{n}\|v\|, \text { for all } n \in \mathbb{Z} .
\end{gathered}
$$

The stable and unstable bundles $E^{s}$ and $E^{u}$ are uniquely integrable and tangent to stable and unstable foliation $\left\{W_{x}^{s}\right\}_{x \in A}$ and $\left\{W_{x}^{u}\right\}_{x \in A}$, respectively, whose leaves are $C^{1}$-smooth.

There exists a $C^{1}$-smooth local coordinate system $(u, s, c)$ in some tubular neighborhood of $A$ in $M$ such that each point $x$ in the tubular neighborhood corresponds uniquely to coordinates $(u, s, c)$ where $c=(I, \phi)$ are the action-angle coordinates in $A, u \in E_{c}^{u}$, and $s \in E_{c}^{s}$. For example, we can write each point $x$ in the neighborhood as $x=\exp _{c}(v)$, where $v \in E_{c}^{u} \oplus E_{c}^{s}$. In Section 4 we will use this local coordinate system to construct windows in a neighborhood of $A$.

3.2. Birkhoff zones of instability. Let $A=[0,1] \times \mathbb{T}^{1} \subseteq \mathbb{R}^{2}$ be an annulus, thought of as a cylinder with the radius coordinate $I \in[0,1]$, and with the angle coordinate $\phi \in \mathbb{R}$ defined modulo $2 \pi$. A $C^{1}$-diffeomorphism $f: A \rightarrow A$, of components $\left.f(I, \phi)=\left(f^{1}(I, \phi), f^{2}(I, \phi)\right)\right)$, is a monotone twist map provided that is area preserving, orientation preserving, boundary preserving, and satisfies $\partial f^{2} / \partial I>0$. 
We recall that a primary torus (essential invariant circle) in the annulus $A$ is a a 1-dimensional torus in $A$ that cannot be homotopically deformed into a point inside the annulus. We review some classical concepts and results regarding twist maps.

Theorem 3.1 ([3, 4, 15]). If $U$ is an $f$-invariant relatively open subset of the annulus $A$, is simply connected in the universal covering space of $A$, contains one of the boundary circles and is disjoint from the other boundary circle, then the boundary of $U$ is a primary invariant torus (an essential invariant circle), and is the graph of some Lipschitz map.

In Section 4.2 we assumed that the invariant tori $\left\{T_{\iota}\right\}_{\iota \in \mathcal{I}}$ are $C^{1}$-smooth. Thus, they are $C^{1}$-smooth graphs of functions in the $\phi$-variable. This means that the action direction $I$ is transverse to each of these tori. Suppose that we have such a torus $T$ that is the graph of a $C^{1}$-smooth function $I=\tau(\phi)$. We can introduce a new system of coordinates $(\bar{I}, \phi)$ in the neighborhood of this torus, where $\bar{I}=$ $I-\tau(\phi)$. Thus the given torus $T$ is characterized by $\bar{I}=0$, and the twist condition $\partial f^{2}(\bar{I}, \phi) / \partial \bar{I}=\partial f^{2}(I, \phi) / \partial I>0$ is satisfied near $T$ in this new coordinate system. In Section 4 we will use this local coordinate system near $T$ to construct certain 2-dimensional windows near $T$ in $A$. In essence, we will construct windows of the type

$$
R^{I, \phi}=\bigcup_{\theta \in R^{\phi}} R_{\theta}^{I},
$$

where $R^{\phi}$ denotes a closed interval in the $\phi$-coordinate, and $R_{\theta}^{I}$ represents a closed line segment of given length and parallel to the $I$-direction, centered at the point in $T$ of $\phi$-coordinate equal to $\theta$. Such a window has a simple description in the $(\bar{I}, \phi)$-local coordinate system, being the product of two intervals in the $\bar{I}$ - and $\phi$-coordinates

$$
R^{I, \phi}=R^{\bar{I}} \times R^{\phi} .
$$

Definition 3.2. A region in the annulus bounded by two primary invariant tori which contains no primary invariant torus in its interior is called a (Birkhoff) zone of instability.

Theorem $3.3([4,33])$. Given a zone of instability bounded by the tori $T_{1}$ and $T_{2}$, for every $\epsilon>0$ there exist a point $p$ and an integer $n>0$ such that $d\left(p, T_{1}\right)<\epsilon$ and $d\left(f^{n}(p), T_{2}\right)<\epsilon$. Moreover, if $f$ is, additionally, topologically transitive on both $T_{1}$ and $T_{2}$, then for every point $z_{1} \in T_{1}$ and every open neighborhood $B_{1}$ of $z_{1}$ in $A$, and for every point $z_{2} \in T_{2}$ and every open neighborhood $B_{2}$ of $z_{2}$ in $A$, there exists a point $p \in B_{1}$ and an integer $K>0$ such that $f^{K}(p) \in B_{2}$.

Later, in Subsection 4.1.4 and in Subsection 4.2.4, we will need a stronger version of the above result, which we state and prove below.

Lemma 3.4. Consider a zone of instability $Z$ bounded by two invariant primary tori $T_{1}$ and $T_{2}$. Assume that the restriction of $f$ to each of these tori is topologically transitive. Let $z_{1} \in T_{1}$ and $z_{2} \in T_{2}$ be a pair of points. Let $c:[0,1] \rightarrow Z$ be a $C^{1}$-curve such that $c(0)=z_{2} \in T_{2}$ and $c(0,1] \subseteq Z$, let $B_{1}$ be an open neighborhood of $z_{1}$ in $Z$, and let $\epsilon>0$. Then there exist $K>0$ and a point $p=c\left(t_{\varepsilon}\right)$ on the curve $c$, where $0<t_{\epsilon}<\epsilon$, such that $f^{-K}(p) \in B_{1}$. 
Proof. Let

$$
V=\operatorname{cl}\left[\bigcup_{K \geq 0} f^{-K}(c[0, \epsilon])\right]
$$

This is a closed set that contains $T_{2}$, since $f$ is topologically transitive on $T_{2}$. Hence $V$ is connected. We also have $f^{-1}(V) \subseteq V$. Assume that $V$ does not intersect the open set $B_{1}$. Since $f$ is topologically transitive on $T_{1}$, it follows that $V$ does not intersect a whole open neighborhood of $T_{1}$ in $Z$. The complementary set $\complement V$ of $V$ in $Z$ is an open set that contains $T_{1}$ and satisfies $f^{-1}(\complement V) \supseteq \complement V$. Let $U$ be the connected component of $\complement V$ that contains $T_{1}$. Since $U$ is covered by the open connected components of $f^{-1}(\complement V)$, and $T_{1} \subseteq U$ is invariant, we must have $f^{-1}(U) \supseteq U$. Since $f$ is area preserving, $f^{-1}(U)=U$. Also, by identifying $Z$ with its universal covering space and collapsing $T_{1}$ to a point, and applying Lemma A.7.8 from [27], we derive that $U$ is simply connected in the covering space of $Z$. The boundary of $U$ cannot meet $T_{2}$, since otherwise it will coincide with $T_{2}$, due to $f$ being topologically transitive on $T_{2}$. By Theorem 3.1, the boundary of $U$ is a primary invariant torus between $T_{1}$ and $T_{2}$, and is the graph of a Lipschitz map. This contradicts that $Z$ is a zone of instability. Therefore, $V$ must intersect the open set $B_{1}$.

We also note that $\left(d f^{-K}\right)_{p}$ is an invertible map, therefore we can always find a vector $v$ at $p$ that is transverse to a certain prescribed direction $w_{2}$ at $p$, whose image $\left(d f^{-K}\right)_{p}(v)$ under $\left(d f^{-K}\right)_{p}$ is a vector that is transverse to a certain prescribed direction $w_{1}$ at $f^{-K}(p)$.

3.3. Sequences of invariant tori. In the statement of Theorem 1.2, a finite sequence of invariant tori $\left\{T_{i_{t}}\right\}_{t}$ from the Cantor family $\left\{T_{\iota}\right\}_{\iota}$, and a finite sequence $\left\{\epsilon_{t}\right\}_{t}$ of positive real numbers are given. We want to prove the existence of an orbit $\left\{z_{t}\right\}_{t}$ whose points are $\left(\epsilon_{t}\right)$-close to $\left\{T_{i_{t}}\right\}_{t}$.

We replace the given sequence $\left\{T_{i_{t}}\right\}_{t}$ with a new finite sequence of tori chosen from $\left\{T_{\iota}\right\}_{\iota}$, as follows. To $\left\{T_{i_{t}}\right\}_{t=0, \ldots, s}$ we first add all the tori $T_{j_{0}}, \ldots, T_{j_{2 m+1}}$ described in $H 3$. We remind the reader that the tori $T_{j_{0}}, T_{j_{1}}, \ldots, T_{j_{2 m+1}}$ are at the boundaries of the Birkhoff zones of instability. Then we inspect each torus $T_{i_{t}}$ that lies in between some $T_{j_{2 k}}$ and $T_{j_{2 k+1}}$, for $k=0, \ldots, m$ : if $T_{i_{t}}$ is an 'interior' torus, in the sense described in the explanatory paragraphs following hypothesis $H 1$, we do nothing; if $T_{i_{t}}$ is not an 'interior' torus, then we replace it by an 'interior' torus within $\left(\epsilon_{t} / 2\right)$ of the original one. Finally, we add additional tori between each pair $T_{j_{2 k}}, T_{j_{2 k+1}}, k=0, \ldots, m$ such that transition chains connecting $T_{j_{2 k+1}}$ with $T_{j_{2 k+2}}$ can be formed; each additional torus is chosen from the 'interior' tori of the Cantor family $\left\{T_{\iota}\right\}_{\iota \in \mathcal{I}}$. This can be done due to hypotheses H1 and H3.a on the Cantor family $\left\{T_{\iota}\right\}_{\iota \in \mathcal{I}}$..

With an abuse of notation, we denote the resulting finite sequence also by $\left\{T_{i_{t}}\right\}_{t=0, \ldots, s}$. The tori $T_{j_{0}}, T_{j_{1}}, \ldots, T_{j_{2 m+1}}$ described in H3 are part of the new sequence $\left\{T_{i_{t}}\right\}_{t=0, \ldots, s}$. The set of indices $\left\{j_{0}, \ldots, j_{2 m+1}\right\}$ is a subset of the set of indices $\left\{i_{0}, \ldots, i_{s}\right\}$.

By construction, if an orbit $(\epsilon / 2)$-shadows this new sequence, then the same orbit $\left(\epsilon_{t}\right)$-shadows the original sequence from the statement of Theorem 1.2. This new sequence $\left\{T_{i_{t}}\right\}_{t}$ satisfies the properties P1, P2, P3.a, P3.b listed below. These 
properties are inherited from the properties H1, H2, H3.a, H3.b of the Cantor family $\left\{T_{\iota}\right\}_{\iota}$.

P1. Each torus $T_{i_{t}}$ with $j_{2 k} \prec i_{t} \prec j_{2 k+1}$ is the limit in the $C^{1}$-topology of a sequence of tori $\left\{T_{\iota_{n}^{\prime}}\right\}_{n}$ from $\left\{T_{\iota}\right\}_{\iota}$, with $\iota_{n}^{\prime} \prec \iota$ and $\iota_{n}^{\prime} \rightarrow i_{t}$ as $n \rightarrow \infty$, and is also the limit in the $C^{1}$-topology of a sequence of tori $\left\{T_{\iota_{n}^{\prime \prime}}\right\}_{n}$ from $\left\{T_{\iota}\right\}_{\iota}$, where $\iota \prec \iota_{n}^{\prime \prime}$ and $\iota_{n}^{\prime \prime} \rightarrow i_{t}$ as $n \rightarrow \infty$.

The tori $T_{i_{t}}$ with $j_{2 k} \prec i_{t} \prec j_{2 k+1}$ were referred in the explanatory paragraphs following hypothesis $H 1$ as 'interior' tori; they are not at the boundary of Birkhoff zones of instability. Note that the sequences of tori $\left\{T_{\iota_{n}^{\prime}}\right\}_{n}$ and $\left\{T_{\iota_{n}^{\prime \prime}}\right\}_{n}$ that approximate $T_{i_{t}}$ are from $\left\{T_{\iota}\right\}_{\iota}$ and not from $\left\{T_{i_{t}}\right\}_{t}$.

P2. We assume that $\left.F\right|_{A}$ is topologically transitive on each torus from $\left\{T_{i_{t}}\right\}_{t}$.

This condition replicates condition $\mathrm{H} 2$.

P3.a. For $k=0, \ldots, m$, for each pair of tori $T_{i_{t}}, T_{i_{t+1}}$ with $j_{2 k} \preceq i_{t} \prec i_{t+1} \preceq$ $j_{2 k+1}, W^{u}\left(T_{i_{t}}\right)$ intersects transversally $W^{s}\left(T_{i_{t+1}}\right)$.

This means that there exist transition chains of tori connecting the upper boundary of one zone of instability to the lower boundary of the next zone of instability.

P3.b. For $k=0, \ldots, m-1$, the region in $A$ between $T_{j_{2 k+1}}$ and $T_{j_{2 k+2}}$ is a Birkhoff zone of instability.

This condition, which replicates condition H3.b, does not prescribe whether or not there exist heteroclinic connections between the boundaries of the Birkhoff zones of instability lying between $T_{j_{2 k+1}}$ and $T_{j_{2 k+2}}$, where $k=0, \ldots, m-1$. The conditions P3.a and P3.b together imply that the new sequence $\left\{T_{i_{t}}\right\}_{t}$ consists in transition chains of tori alternating with 'large gaps', which are Birkhoff zones of instability. Each pair of tori $T_{j_{2 k+1}}$ and $T_{j_{2 k+2}}$ at the boundary of a large gap are part of the new sequence.

3.4. Heteroclinic points. Consider the sequence of invariant tori $\left\{T_{i_{t}}\right\}_{t}$ obtained in Section 3.3. If $W^{u}\left(T_{i_{t}}\right)$ intersects $W^{s}\left(T_{i_{t+1}}\right)$ transversally, then the intersection is 0-dimensional; we choose and fix a heteroclinic point $q_{t}$ in the intersection. If $q_{t}$ is in the intersection of $W^{u}\left(T_{i_{t}}\right)$ with $W^{s}\left(T_{i_{t+1}}\right)$, then, due to normal hyperbolicity (see Section 3.1), there exist unique points $p_{t}^{\prime} \in T_{i_{t}}$ and $p_{t+1} \in T_{i_{t+1}}$ such that $q_{t} \in W^{u}\left(p_{t}^{\prime}\right) \cap W^{s}\left(p_{t+1}\right)$.

3.5. Construction of correctly aligned windows about a heteroclinic point. We consider a pair of $C^{1}$-smooth invariant tori $T_{i_{t}}$ and $T_{i_{t+1}}$ in $A$ that have a transverse heteroclinic intersection at $q_{t}$. We are going to construct a pair of windows $W_{t}^{\prime}$ and $W_{t+1}$ near $q_{t}$ that are correctly aligned under the identity map.

Since $W^{u}\left(T_{i_{t}}\right)$ is transverse to $W^{s}\left(T_{i_{t+1}}\right)$, there exists a local coordinate system $h_{t}: U_{t} \rightarrow \mathbb{R}^{2 n+2} \simeq T_{q_{t}} M$ in a neighborhood $U_{t} \subseteq M$ of $q_{t}$, given by $h_{t}(p)=(x, y) \in$ $\mathbb{R}^{n+1} \times \mathbb{R}^{n+1}$, such that the branch of $W^{u}\left(T_{i_{t}}\right)$ in $U_{t}$ that contains $q_{t}$ is given by $y=0$, and the branch of $W^{s}\left(T_{i_{t+1}}\right)$ in $U_{t}$ that contains $q_{t}$ is given by $x=0$.

In this local coordinate system, we let $W_{t}^{\prime}$ be the window in $U_{t}$ given in local coordinates by $\left[a_{t}^{\prime}, b_{t}^{\prime}\right]^{n+1} \times\left[c_{t}^{\prime}, d_{t}^{\prime}\right]^{n+1}$, and $W_{t+1}$ be the window in $U_{t}$ given in local coordinates by $\left[a_{t+1}, b_{t+1}\right]^{n+1} \times\left[c_{t+1}, d_{t+1}\right]^{n+1}$. We define the exit set of $W_{t}^{\prime}$ by $\left(W_{t}^{\prime}\right)^{-}=\left(h_{t}\right)^{-1}\left(\partial\left(\left[a_{t}^{\prime}, b_{t}^{\prime}\right]^{n+1}\right) \times\left[c_{t}^{\prime}, d_{t}^{\prime}\right]^{n+1}\right)$, and the exit set of $W_{t+1}$ by $\left(W_{t+1}\right)^{-}=$ $\left(h_{t}\right)^{-1}\left(\partial\left(\left[a_{t+1}, b_{t+1}\right]^{n+1}\right) \times\left[c_{t+1}, d_{t+1}\right]^{n+1}\right)$. We choose $a_{t}^{\prime}<a_{t+1}<0<b_{t+1}<b_{t}^{\prime}$ and $c_{t+1}<c_{t}^{\prime}<0<d_{t}^{\prime}<d_{t+1}$ in order to ensure the correct alignment of $W_{t}^{\prime}$ with $W_{t+1}$ with respect to the identity map, in local coordinates. These inequalities imply the correct alignment conditions (i) and (ii) of Definition 2.2. See Figure 


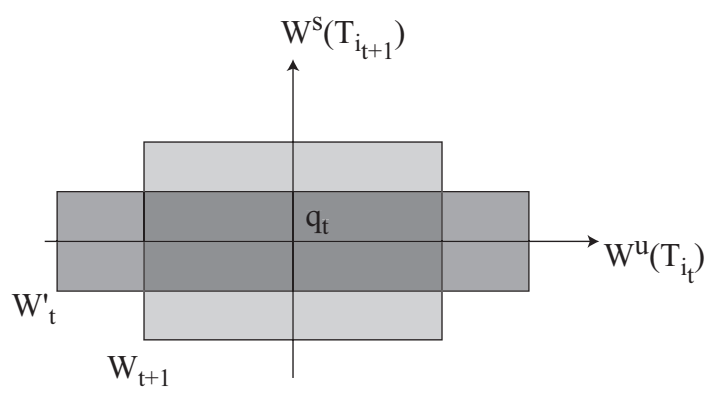

FiguRE 5. Construction of correctly aligned windows under the identity mapping, near the transverse intersection of the invariant manifolds.

5. (Another way to argue this correct alignment would be based on Proposition 2.4 and on Proposition 2.6.) Therefore, the windows $W_{t}^{\prime}$ and $W_{t+1}$ are correctly aligned under the identity, since their counterparts in $\mathbb{R}^{2 n+2}$ are correctly aligned under the identity. At this point, we impose no additional requirements on the size of the windows. In the sequel we will refine and adjust these windows according to their behavior under dynamics.

Let us note that each window $W_{t}^{\prime}$ with $W_{t+1}$ is foliated by leaves of the type $y=$ const. and also by leaves of the type $x=$ const. These two types of leaves are transverse to one another. We emphasize that these leaves, with the exception of $y=0$ and $x=0$, which correspond to the unstable manifold of $T_{i_{t}}$ and to the stable manifold of $T_{i_{t+1}}$ respectively, are not necessarily invariant under the dynamics.

3.6. Construction of correctly aligned windows by the transversalitytorsion mechanism. Let us consider a pair of correctly aligned windows $W_{t}^{\prime}$ and $W_{t+1}$ about $q_{t}$, as above, where $q_{t} \in W^{u}\left(p_{t}^{\prime}\right) \cap W^{s}\left(p_{t+1}\right)$ with $p_{t}^{\prime} \in T_{i_{t}}$ and $p_{t+1} \in T_{i_{t+1}}$. We will first describe the behavior of the exit and entry directions of $W_{t}^{\prime}$ when this window undergoes backward iterations, and then the behavior of the exit and entry directions of $W_{t+1}$ when this window undergoes forward iterations.

We take a negative iterate $F^{-M_{t}}\left(W_{t}^{\prime}\right)$ so that $F^{-M_{t}}\left(q_{t}\right)$ is at a distance of less than $\left(\epsilon_{t} / 2\right)$ from $F^{-M_{t}}\left(p_{t}^{\prime}\right) \in T_{i_{t}}$, where the distance is measured along $W^{u}\left(T_{i_{t}}\right)$. The exit set of the window $W_{t}^{\prime}$ is made of the union of the boundaries of the leaves $\{y=$ const. $\} \cap W_{t}^{\prime}$; the entry set is made of the union of the boundaries of the leaves $\{x=$ const. $\} \cap W_{t}^{\prime}$. The tangent space to $W^{u}\left(T_{i_{t}}\right)$ at $q_{t}$ is spanned by $n$ vectors $\left(v_{1}^{\prime}, \ldots, v_{n}^{\prime}\right)$ tangent to $W^{u}\left(p_{t}^{\prime}\right)$ at $q_{t}$ and an $(n+1)$-th vector $v_{n+1}^{\prime}$ transverse to the subspace spanned by these $n$-vectors (relative to $T_{q_{t}} W^{u}\left(T_{i_{t}}\right)$ ). Under the derivative of $F^{-M_{t}}$, the subspace spanned by the vectors $\left(v_{1}^{\prime}, \ldots, v_{n}^{\prime}\right)$ will go to the tangent space of $W^{u}\left(F^{-M_{t}}\left(p_{t}^{\prime}\right)\right)$ at $F^{-M_{t}}\left(q_{t}\right)$, and will approach the tangent space of $W^{u}\left(F^{-M_{t}}\left(p_{t}^{\prime}\right)\right)$ at $F^{-M_{t}}\left(p_{t}^{\prime}\right)$ as $M_{t}$ increases. For any finite number of iterations $M_{t}$, the image of $v_{n+1}^{\prime}$ under the derivative of $F^{-M_{t}}$ will remain tangent to $W^{u}\left(T_{i_{t}}\right)$ and transverse to $W^{u}\left(F^{-M_{t}}\left(p_{t}^{\prime}\right)\right)$. On the other hand, the tangent space to $W^{s}\left(T_{i_{t+1}}\right)$ at $q_{t}$ is spanned by $(n+1)$ vectors $\left(w_{1}^{\prime}, \ldots, w_{n+1}^{\prime}\right)$; we can choose these vectors such that, under the derivative of $F^{-M_{t}}$, the subspace spanned by $\left(w_{1}^{\prime}, \ldots, w_{n}^{\prime}\right)$ will approach the tangent space of $W^{s}\left(F^{-M_{t}}\left(p_{t}^{\prime}\right)\right)$ at $F^{-M_{t}}\left(p_{t}^{\prime}\right)$, while the image of $w_{n+1}$ will not grow exponentially in length and will remain transverse to $W^{u}\left(T_{i_{t}}\right)$. This 
follows from the transversality of the intersection between $W^{u}\left(T_{i_{t}}\right)$ and $W^{s}\left(T_{i_{t+1}}\right)$, and from normal hyperbolicity. The topological linearization (Hartman-Grobman type result) of Pugh-Shub could be used to see the above statements (see [37]).

The difference between choosing $\left(v_{1}, \ldots, v_{n+1}\right)$ and choosing $\left(w_{1}, \ldots, w_{n+1}\right)$ is that we we cannot specify whether or not the vectors $w_{1}, \ldots, w_{n}$ that approach the stable directions correspond to vectors tangent to $W^{s}\left(p_{t+1}\right)$ at $q_{t}$. We emphasize that we are not concerned with the behavior of these vectors in the limit, but only after a finite number of iterations. Thus, the exit directions of the window $F^{-M_{t}}\left(W_{t}^{\prime}\right)$ at $F^{-M_{t}}\left(q_{t}\right)$ correspond to the images of the vectors $v_{1}^{\prime}, \ldots, v_{n+1}^{\prime}$ under the derivative of $F^{-M_{t}}$, while the entry directions correspond to the images of the vectors $w_{1}^{\prime}, \ldots, w_{n+1}^{\prime}$ under the derivative of $F^{-M_{t}}$. When restricted to the center directions, the exit direction is given by $D_{q_{t}}\left(F^{-M_{t}}\right)\left(v_{n+1}^{\prime}\right)$ which is tangent to $W^{u}\left(F^{-M_{t}}\left(p_{t}^{\prime}\right)\right)$, and the entry direction is given by $D_{q_{t}}\left(F^{-M_{t}}\right)\left(w_{n+1}^{\prime}\right)$ which is transverse to $W^{u}\left(F^{-M_{t}}\left(p_{t}^{\prime}\right)\right)$. Thus, the projection onto the $(I, \phi)$-coordinates of the 2-dimensional section in $F^{-M_{t}}\left(W_{t}^{\prime}\right)$, through $F^{-M_{t}}\left(q_{t}\right)$, corresponding to $D_{q_{t}}\left(F^{-M_{t}}\right)\left(v_{n+1}^{\prime}\right)$ and $D_{q_{t}}\left(F^{-M_{t}}\right)\left(w_{n+1}^{\prime}\right)$ is a topological parallelogram $R$ that crosses $T_{i_{t}}$. The exit of this topological rectangle consists of two curves transverse to $T_{i_{t}}$. We can construct a rectangle $R$ in $A$, of the type $R^{I, \phi}=\bigcup_{\theta \in R^{\phi}} R_{\theta}^{I}$ (as in (3.2)), with the exit set in the $\phi$-direction, which is correctly aligned with the projected topological rectangle from above under identity. See Figure 8 (right). Using continuity, by choosing the initial window $W_{t}^{\prime}$ sufficiently small, choosing $R^{I, \phi}$ sufficiently wide in the $\phi$-direction and sufficiently narrow in the $I$-direction, and taking its product by a rectangle $R^{u, s}$ in the $(u, s)$-coordinates, we can construct a new window $\widehat{W}_{t}=R^{I, \phi} \times R^{u, s}$, centered at $F^{-M_{t}}\left(p_{t}^{\prime}\right)$, whose exit directions at $F^{-M_{t}}\left(p_{t}^{\prime}\right)$ are precisely given by the angle direction $\phi$ along $T_{i_{t}}$ and by the unstable directions at the points on $T_{i_{t}}$, and whose entry directions are precisely given by the action direction $I$ and by the stable directions at the points on $T_{i_{t}}$, such that $\widehat{W}_{t}$ is correctly aligned with $F^{-M_{t}}\left(W_{t}^{\prime}\right)$ under the identity map, or, equivalently, $\widehat{W}_{t}$ is correctly aligned with $W_{t}^{\prime}$ under $F^{M_{t}}$. See Remark 2.7.

Similarly, we take a positive iterate $F^{N_{t+1}}\left(W_{t+1}\right)$ so that $F^{N_{t+1}}\left(q_{t}\right)$ is at a distance less than $\left(\epsilon_{t+1} / 2\right)$ from $F^{N_{t+1}}\left(p_{t+1}\right) \in T_{i_{t+1}}$, where the distance is measured along $W^{s}\left(T_{i_{t+1}}\right)$. In the tangent space to $W^{u}\left(T_{i_{t}}\right)$ at $q_{t}$ we can choose a basis $\left(v_{1}, \ldots, v_{n+1}\right)$ such that, the image of the subspace spanned by $\left(v_{1}, \ldots, v_{n}\right)$ under the derivative of $F^{N_{t+1}}$ will approach the tangent space of $W^{u}\left(F^{N_{t+1}}\left(p_{t+1}\right)\right)$ at $F^{N_{t+1}}\left(p_{t+1}\right)$, while the image of the $v_{n+1}$ under the derivative of $F^{N_{t+1}}$ will not grow exponentially in length and will remain transverse to $W^{s}\left(T_{i_{t+1}}\right)$. The tangent space to $W^{s}\left(T_{i_{t+1}}\right)$ at $q_{t}$ has a basis of vectors $\left(w_{1}, \ldots, w_{n+1}\right)$ such that, the image of the subspace spanned by $\left(w_{1}, \ldots, w_{n}\right)$ under the derivative of $F^{N_{t+1}}$ will approach the tangent space of $W^{s}\left(F^{N_{t+1}}\left(p_{t+1}\right)\right.$ at $F^{N_{t+1}}\left(p_{t+1}\right)$, while the image of $w_{n+1}$ under the derivative of $F^{N_{t+1}}$ will remain tangent to $W^{s}\left(T_{i_{t+1}}\right)$. Thus, the exit directions of $F^{N_{t+1}}\left(W_{t+1}\right)$ at $F^{N_{t+1}}\left(q_{t}\right)$ correspond to the images of the vectors $v_{1}, \ldots, v_{n}, v_{n+1}$ under the derivative of $F^{N_{t+1}}$, while the entry directions correspond to the images of the vectors $w_{1}, \ldots, w_{n}, w_{n+1}$ under the derivative of $F^{N_{t+1}}$. Restricted to the center directions, the exit direction is given by $D_{q_{t}}\left(F^{N_{t+1}}\right)\left(v_{n+1}\right)$, and the entry direction is given by $D_{q_{t}}\left(F^{N_{t+1}}\right)\left(w_{n+1}\right)$. Thus, the projection in $(I, \phi)$-coordinates of the 2-dimensional section in $F^{N_{t+1}}\left(W_{t+1}\right)$, through $F^{N_{t+1}}\left(q_{t}\right)$, corresponding to $D_{q_{t}}\left(F^{N_{t+1}}\right)\left(v_{n+1}\right)$ and $D_{q_{t}}\left(F^{N_{t+1}}\right)\left(w_{n+1}\right)$ is a topological rectangle that crosses $T_{i_{t+1}}$; its exit set consist of two curves, one on each side of $T_{i_{t+1}}$. 


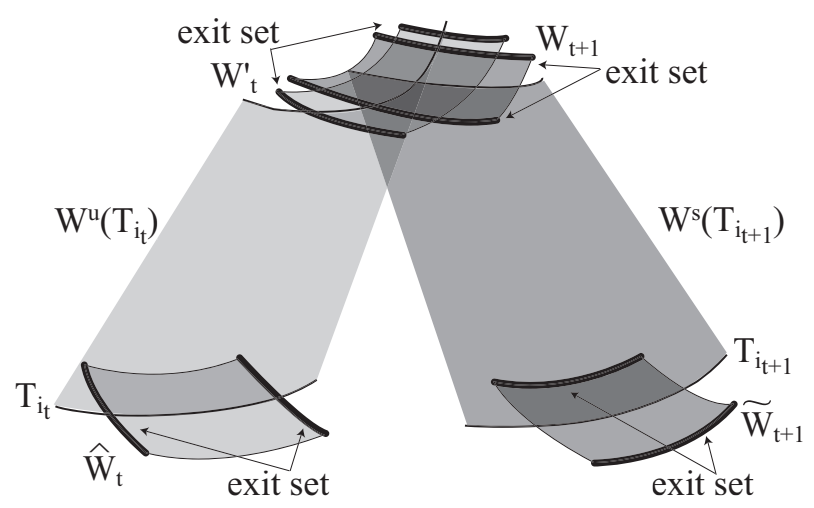

FiguRE 6. The transversality-torsion mechanism: the exit sets of the windows propagated near the annulus have reverse orientations with respect to the center directions.

We can we can construct a rectangle $R^{I, \phi}$ in $A$, with the exit set in the $I$-direction, that is correctly aligned with the projected topological rectangle from above under identity. See Figure 7 (right). Using continuity, by choosing $W_{t+1}$ sufficiently small, and choosing $R^{I, \phi}$ sufficiently narrow in the $\phi$-direction and sufficiently wide in the $I$-direction, we can construct a new window of product type $\widetilde{W}_{t+1}=R^{I, \phi} \times R^{u, s}$, centered at $F^{N_{t+1}}\left(p_{t+1}\right)$, whose exit directions at $F^{N_{t+1}}\left(p_{t+1}\right)$ are precisely given by the action direction $I$ and by the unstable directions at the points on $T_{i_{t+1}}$, and whose entry directions are precisely given by the angle direction $\phi$ and by the stable directions at the points on $T_{i_{t+1}}$, such that $F^{N_{t}}\left(W_{t+1}\right)$ is correctly aligned with $\widetilde{W}_{t+1}$ under the identity map, or, equivalently, $W_{t+1}$ is correctly aligned with $\widetilde{W}_{t+1}$ under $F^{N_{t+1}}$.

If we focus on the center components of the exit directions of $\widehat{W}_{t}$ and of $\widetilde{W}_{t+1}$, we notice that the exit direction of $\widehat{W}_{t}$ points in the $\phi$-direction and the entry direction points in the $I$-direction, while the exit direction of $\widetilde{W}_{t+1}$ points in the $I$-direction and the entry direction points in the $\phi$-direction. We will refer to this mechanism of swapping the exit and entry directions between these two windows by the term of transversality-torsion, inspired from [8]. With respect to the hyperbolic directions, the exit sets of both $\widehat{W}_{t}$ and $\widetilde{W}_{t+1}$ point in the unstable directions. See Figure 6.

\section{Proof of the MAIN THEOREM}

Given a sequence $\left\{T_{i_{t}}\right\}_{t=0, \ldots, s}$ as described in Subsection 3.3, we construct inductively a sequence of windows $\left\{\widetilde{W}_{t}\right\}_{t=0, \ldots, s}$ with $\widetilde{W}_{t}$ near the torus $T_{i_{t}}$, such that $\widetilde{W}_{t}$ and $\widetilde{W}_{t+1}$ are linked by a sequence of correctly aligned windows for each $t$. The strategy is to begin with a pair of correctly aligned windows about the first heteroclinic connection, and to propagate the construction of correctly aligned windows forward, along transition chains and across zones of instability. Whenever we will cross a zone of instability, we will need to go back and adjust some of the correctly aligned windows constructed prior to that step. The inductive process through which the windows are constructed and adjusted is described below. 
4.1. Initial step. Starting with the first heteroclinic connection, we construct a finite sequence of correctly aligned windows along the first transition chain and across the first large gap.

4.1.1. Construction of correctly aligned windows about a heteroclinic point. We first consider the heteroclinic intersection of $W^{u}\left(T_{i_{0}}\right)$ with $W^{s}\left(T_{i_{1}}\right)$ at $q_{0}$ (see Subsection 3.4). About $q_{0}$, we construct a pair of windows $W_{0}^{\prime}$ and $W_{1}$ that are correctly aligned under the identity mapping, following the procedure described in Subsection 3.5.

4.1.2. Construction of correctly aligned windows by the transversality-torsion mechanism. We choose the sizes of the windows $W_{0}^{\prime}$ and $W_{1}$ such that the behavior of the positive iterates of $W_{1}$ and of the negative iterates of $W_{0}^{\prime}$ is as prescribed below.

We take a positive iterate $F^{N_{1}}\left(W_{1}\right)$ so that the distance from any point in $F^{N_{1}}\left(W_{1}\right)$ to $T_{i_{1}}$, measured with respect to the $(I, s)$-coordinates, is less than $\left(\epsilon_{1} / 2\right)$. Hence $F^{N_{1}}\left(q_{0}\right)$ is $\left(\epsilon_{1} / 2\right)$-close to $F^{N_{1}}\left(p_{1}\right) \in T_{i_{1}}$. We construct a window $\widetilde{W}_{1}$ centered at the point $F^{N_{1}}\left(p_{1}\right)$ in $T_{i_{1}}$ such that $W_{1}$ is correctly aligned with $\widetilde{W}_{1}$ under $F^{N_{1}}$, and the distance from any point in $\widetilde{W}_{1}$ to $T_{i_{1}}$, measured with respect to the $(u, s, I)$-coordinates, is less than $\left(\epsilon_{1} / 2\right)$. To describe this window more precisely, let $(\bar{I}, \phi)$ be a local coordinate system near $T_{i_{1}}$, as described in Subsection 3.2. We construct the window $\widetilde{W}_{1}$ as a product of 4 topological rectangles (i.e., homeomorphic copies of cartesian rectangles), corresponding to the $u, s, \phi, \bar{I}$ coordinates, respectively:

$$
\widetilde{W}_{1}=\widetilde{R}_{1}^{u} \times \widetilde{R}_{1}^{s} \times \widetilde{R}_{1}^{\phi} \times \widetilde{R}_{1}^{\bar{I}}
$$

with the rectangles $\widetilde{R}_{1}^{u}$ and $\widetilde{R}_{1}^{s}$ centered at 0 , and the exit set given by

$$
\widetilde{W}_{1}^{-}=\left[\partial\left(\widetilde{R}_{1}^{u}\right) \times \widetilde{R}_{1}^{s} \times \widetilde{R}_{1}^{\phi} \times \widetilde{R}_{1}^{\bar{I}}\right] \cup\left[\widetilde{R}_{1}^{u} \times \widetilde{R}_{1}^{s} \times \widetilde{R}_{1}^{\phi} \times \partial\left(\widetilde{R}_{1}^{\bar{I}}\right)\right] .
$$

The exit set of $\widetilde{W}_{1}$ is in the $(u, \bar{I})$-direction, in accordance with the transversalitytorsion mechanism (Subsection 3.6). Moreover, we require that the rectangle $\{0\} \times\{0\} \times \widetilde{R}_{1}^{\phi} \times \widetilde{R}_{1}^{\bar{I}}$, corresponding to the intersection between $\widetilde{W}_{1}$ and the annulus $A$, falls in between some invariant tori $T_{\iota_{1}^{\prime}}, T_{\iota_{1}^{\prime \prime}}$ from the Cantor family $\left\{T_{\iota}\right\}_{\iota \in \mathcal{I}}$, with $\iota_{1}^{\prime}<i_{1}<\iota_{1}^{\prime \prime}$, and $\iota_{1}^{\prime \prime}-i_{1}<\epsilon_{1} / 2$ and $i_{1}-\iota_{1}^{\prime}<\epsilon_{1} / 2$. Such a choice is possible due to condition $P 1$. The exit set corresponding to the rectangle $\{0\} \times\{0\} \times \widetilde{R}_{1}^{\phi} \times \widetilde{R}_{1}^{\bar{I}}$, which is given by $\{0\} \times\{0\} \times \widetilde{R}_{1}^{\phi} \times \partial\left(\widetilde{R}_{1}^{\bar{I}}\right)$, is required to have its components lying on the tori $T_{\iota_{1}^{\prime}}$ and $T_{\iota_{1}^{\prime \prime}}$. See Figure 7 , for the case $t=1$.

The condition on $\widetilde{W}_{1}$ implies that size the of $\widetilde{W}_{1}$ measured with respect to the $\bar{I}$-coordinate is sufficiently small. Hence, in order to have $W_{1}$ correctly aligned with $\widetilde{W}_{1}$ under $F^{N_{1}}$, we need the window $W_{1}$ be sufficiently small in the exit direction, thus we need to adjust the window $W_{0}^{\prime}$ such that it is sufficiently small in the exit direction. This completes for now the construction of the pair of windows $W_{0}^{\prime}$ and $W_{1}$ about $q_{0}$, and of the window $\widetilde{W}_{1}$ about $\widetilde{T}_{i_{1}}$.

We then take a negative iterate $F^{-M_{0}}\left(W_{0}^{\prime}\right)$ so that the distance from any point in $F^{-M_{0}}\left(W_{0}^{\prime}\right)$ to $T_{i_{0}}$, measured with respect to the $(I, u)$-coordinates, is less than $\left(\epsilon_{0} / 2\right)$. Hence $F^{-M_{0}}\left(q_{0}\right)$ is $\left(\epsilon_{0} / 2\right)$-close to $F^{-M_{0}}\left(p_{0}^{\prime}\right) \in T_{i_{0}}$. We construct a window $\widehat{W}_{0}$ about $T_{i_{0}}$, centered at $F^{-M_{0}}\left(p_{0}^{\prime}\right)$, such that $\widehat{W}_{0}$ is correctly aligned with $W_{0}^{\prime}$ under $F^{M_{0}}$, and the distance from any point in $\widehat{W}_{0}$ to $T_{i_{0}}$, measured with respect to the $(u, s, I)$-coordinates, is less than $\left(\epsilon_{0} / 2\right)$. Again, we let $(\bar{I}, \phi)$ be a local coordinate system near $T_{i_{0}}$, as described in Subsection 3.2. The window $\widehat{W}_{0}$ is also 

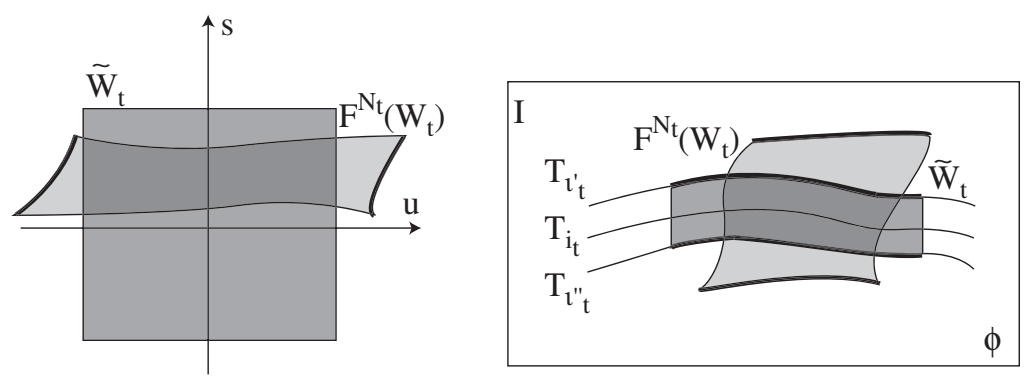

FiguRE 7. Construction of correctly aligned windows near $T_{i_{t}}$, in the case of no gap - sections along the $(u, s)$-coordinates (left), and sections along the $(\phi, I)$-coordinates (right); the exit sets of the sections are marked by thicker lines.

defined as a a product of 4 topological rectangles, corresponding to the $u, s, \phi, \bar{I}$ coordinates, respectively:

$$
\widehat{W}_{0}=\widehat{R}_{0}^{u} \times \widehat{R}_{0}^{s} \times \widehat{R}_{0}^{\phi} \times \widehat{R}_{0}^{\bar{I}}
$$

with the rectangles $\widehat{R}_{0}^{u}$ and $\widehat{R}_{0}^{s}$ centered at 0 , and the exit set given by

$$
\widehat{W}_{0}^{-}=\left[\partial\left(\widehat{R}_{0}^{u}\right) \times \widehat{R}_{0}^{s} \times \widehat{R}_{0}^{\phi} \times \widehat{R}_{0}^{\bar{I}}\right] \cup\left[\widehat{R}_{0}^{u} \times \widehat{R}_{0}^{s} \times \partial\left(\widehat{R}_{0}^{\phi}\right) \times \widehat{R}_{0}^{\bar{I}}\right] .
$$

Since this is the very first step of the construction, we de not require additional conditions on $\widehat{W}_{0}$. For this initial step we let $\widetilde{W}_{0}:=\widehat{W}_{0}$, but we note that in the further steps we will construct windows $\widetilde{W}_{t}$ and $\widehat{W}_{t}$ about $T_{i_{t}}$ which will be different from one another. This completes the construction of the first window $\widetilde{W}_{0}$ about $T_{i_{0}}$.

4.1.3. Construction of correctly aligned windows along the first transition chain. We propagate the construction of correctly aligned windows along the first transition chain that starts at $T_{\iota_{0}}=T_{i_{0}}=T_{j_{0}}$ and ends at $T_{j_{1}}$, the lower boundary of the first large gap. Recall from Subsection 3.3 that $T_{j_{1}}$ is a part of the sequence of tori that we want to shadow.

We start with the already constructed window $\widetilde{W}_{1}$ about $T_{i_{1}}$. We have that $\widetilde{W}_{1} \cap A$ is a rectangle, in the $(\phi, \bar{I})$-coordinates about $T_{i_{1}}$, that falls in between two invariant tori $T_{\iota_{1}^{\prime}}, T_{\iota_{1}^{\prime \prime}}$ with $\iota_{1}^{\prime}<i_{1}<\iota_{1}^{\prime \prime}$.

Consider the next heteroclinic intersection of $W^{u}\left(T_{i_{1}}\right)$ with $W^{s}\left(T_{i_{2}}\right)$ at $q_{1}$. We construct a pair of windows $W_{1}^{\prime}$ and $W_{2}$ about $q_{1}$ that are correctly aligned under the identity mapping, as described in Subsection 3.6. We construct the windows $\widehat{W}_{1}$ about $T_{i_{1}}$, and $\widehat{W}_{2}$ about $T_{i_{2}}$ such that $\widehat{W}_{1}$ is correctly aligned with $W_{1}^{\prime}$ under some power $F^{M_{1}}$ of the map $F$, and $W_{2}$ is correctly aligned with $\widetilde{W}_{2}$ under some power $F^{N_{2}}$ of $F$.

Similarly to the construction of $\widehat{W}_{0}$ in Subsection 4.1 .2 , the window $\widehat{W}_{1}$ is centered at the point $F^{-M_{1}}\left(p_{1}^{\prime}\right)$ in $T_{i_{1}}$, and the $(u, s, I)$-distance from any point in $\widehat{W}_{1}$ to $T_{i_{1}}$ is less than $\left(\epsilon_{1} / 2\right)$. The window $\widehat{W}_{1}$ is also defined as a a product of 4 topological rectangles, corresponding to the $u, s, \phi, \bar{I}$ coordinates near $T_{i_{1}}$ :

$$
\widehat{W}_{1}=\widehat{R}_{1}^{u} \times \widehat{R}_{1}^{s} \times \widehat{R}_{1}^{\phi} \times \widehat{R}_{1}^{\bar{I}}
$$



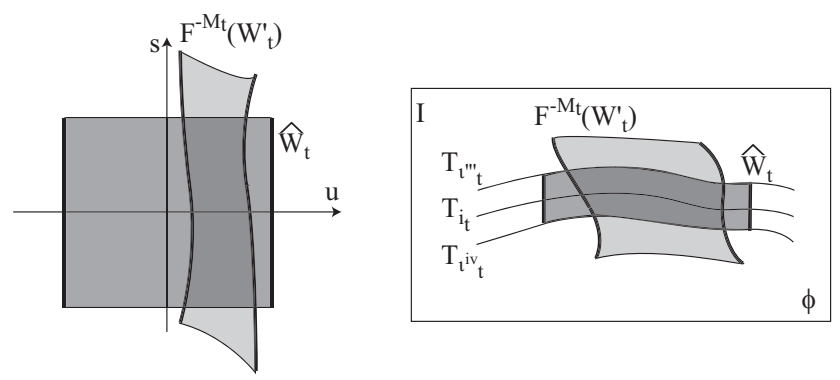

FiguRE 8. Construction of correctly aligned windows near $T_{i_{t}}$, in the case of no gap - sections along the $(u, s)$-coordinates (left), and sections along the $(\phi, I)$-coordinates (right); the exit sets of the sections are marked by thicker lines.

with the rectangles $\widehat{R}_{1}^{u}$ and $\widehat{R}_{1}^{s}$ centered at 0 , and the exit set given by

$$
\widehat{W}_{1}^{-}=\left[\partial\left(\widehat{R}_{1}^{u}\right) \times \widehat{R}_{1}^{s} \times \widehat{R}_{1}^{\phi} \times \widehat{R}_{1}^{\bar{I}}\right] \cup\left[\widehat{R}_{1}^{u} \times \widehat{R}_{1}^{s} \times \partial\left(\widehat{R}_{1}^{\phi}\right) \times \widehat{R}_{1}^{\bar{I}}\right] .
$$

The exit set of $\widehat{W}_{1}$ is in the $(u, \phi)$-directions, in accordance with the transversalitytorsion mechanism (Subsection 3.6). Moreover, we require that the rectangle $\{0\} \times\{0\} \times \widehat{R}_{1}^{\phi} \times \widehat{R}_{1}^{\bar{I}}$, which corresponds to $\widehat{W}_{1} \cap A$, falls in between some invariant tori $T_{\iota_{1}^{\prime \prime \prime}}, T_{\iota_{1}^{i v}}$ from the Cantor family $\left\{T_{\iota}\right\}_{\iota \in \mathcal{I}}$, with $\iota_{1}^{\prime \prime \prime}<\iota_{1}^{\prime}<i_{1}<\iota_{1}^{\prime \prime}<\iota_{1}^{i v}$, and $\iota_{1}^{i v}-i_{1}<\epsilon_{1} / 2$ and $i_{1}-\iota_{1}^{\prime \prime \prime}<\epsilon_{1} / 2$, where $\iota_{1}^{\prime}$ and $\iota_{1}^{\prime \prime}$ are those from Subsection 4.1.2. We require that the entry set of this rectangle, which is given by $\{0\} \times\{0\} \times \widehat{R}_{1}^{\phi} \times \partial\left(\widehat{R}_{1}^{\bar{I}}\right)$ has its components lying on the tori $T_{\iota_{1}^{\prime \prime \prime}}$ and $T_{\iota_{1}^{i v}}$. Such a choice of tori is possible due to condition P1. See Figure 8 , for the case $t=1$.

Now we would like to align the windows $\widetilde{W}_{1}$, described in the previous step, to the window $\widehat{W}_{1}$. We will first align the rectangles $\{0\} \times\{0\} \times \widetilde{R}_{1}^{\phi} \times \widetilde{R}_{1}^{\bar{I}}$ with $\{0\} \times\{0\} \times \widehat{R}_{1}^{\phi} \times \widehat{R}_{1}^{\bar{I}}$. Both rectangles have their centers on $T_{i_{1}}$. The first rectangle has its exit set lying on $T_{\iota_{1}^{\prime}}$ and $T_{\iota_{1}^{\prime \prime}}$, while the second rectangle has its entry set lying on $T_{\iota_{1}^{\prime \prime \prime}}$ and $T_{\iota_{1}^{i v}}$; the last two tori are contained within the strip bounded by the first two tori. The exit direction of the first rectangle is in the $\bar{I}$-direction, while the exit direction of the second rectangle is in the $\phi$-direction. Since the torus $T_{i_{1}}$ is topologically transitive (condition P2), and since the restriction of $F$ to $A$ is a twist map, there exists a power $F^{K_{t}}$ of $F$ which shears $\{0\} \times\{0\} \times \widetilde{R}_{1}^{\phi} \times \widetilde{R}_{1}^{\bar{I}}$ across $\{0\} \times\{0\} \times \widehat{R}_{1}^{\phi} \times \widehat{R}_{1}^{\bar{I}}$ in a manner that is correctly aligned. We note that we need to have the center $F^{K_{1}}\left(F^{N_{1}}\left(p_{1}\right)\right)$ of $\widetilde{W}_{1} \cap A$ at most $\left(\epsilon_{1} / 2\right)$-apart from the center $F^{-M_{1}}\left(p_{1}^{\prime}\right)$ of $\widehat{W}_{1} \cap A$. See Figure 9 , for the case $t=1$. We emphasize that this correct alignment argument is strictly a 2-dimensional one, since it is based on the fact that the invariant tori separate the annulus in disconnected parts. After this correct alignment of the sections $\widetilde{W}_{1} \cap A$ and $\widehat{W}_{1} \cap A$ is ensured, we adjust the sizes of the windows $\widetilde{W}_{1}$ and $\widehat{W}_{1}$ to be small enough in the hyperbolic directions such that for each pair of $(u, s)$-coordinates, the corresponding slices through $\widetilde{W}_{1}$ and $\widehat{W}_{1}$ are correctly aligned under $F^{K_{1}}$. This way, by Proposition 2.6, we obtain that $\widetilde{W}_{1}$ and $\widehat{W}_{1}$ are correctly aligned under $F^{K_{1}}$. Adjustments in size in the $(u, s)$-directions of 


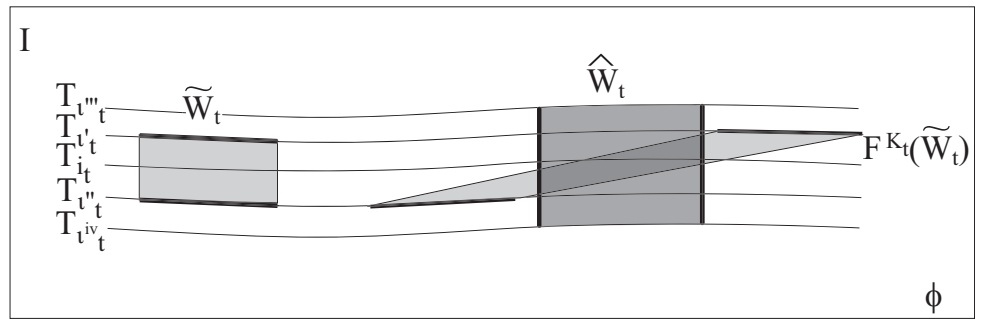

Figure 9. Construction of correctly aligned windows along $T_{i_{t}}$, in the case of no gap - sections along the $(I, \phi)$-coordinates; the exit sets of the sections are marked by thicker lines.

$\widetilde{W}_{1}$ and $\widehat{W}_{1}$ dictate corresponding adjustments in size for all previously constructed windows, and also for $W_{1}^{\prime}$ and $W_{2}$.

From this point on, we continue, in a similar fashion, the construction of windows

$$
\widetilde{W}_{0}, W_{0}^{\prime}, W_{1}, \widetilde{W}_{1}, \widehat{W}_{1}, \ldots, \widetilde{W}_{t_{1}-1}, \widehat{W}_{t_{1}-1}, W_{t_{1}-1}^{\prime}, W_{t_{1}}, \widetilde{W}_{t_{1}},
$$

where $W_{t_{1}}$ is about the torus $T_{i_{t_{1}}}=T_{j_{1}}$, the lower boundary of the first large gap, such that any two consecutive windows in the sequence are correctly aligned under identity or under some power $F^{N_{t}}, F^{K_{t}}, F^{M_{t}}$ of $F$. Each new step of the construction will require the uniform revision of size in the $(u, s)$-directions of the windows constructed up to that point, but neither the revision of the location of the centers of the windows and of the sections with the annulus $A$, nor the revision of the number of iterates of $F$ that it takes to achieve each particular correct alignment. It is quite important to remark that these revisions do not involve the sections of the windows by the annulus $A$, nor their particular positioning between invariant tori from the Cantor family; thus the number of iterates $K_{t}$ that it takes to achieve correct alignment of the sections of the windows by $A$ at each step is not affected by the revisions. Also, the number of iterates $M_{t}$ and $N_{t+1}$ that it takes to bring the heteroclinic point $q_{t}$ sufficiently close to the corresponding tori only depends on the distances from $q_{t}$ to the corresponding tori, and not on the sizes of the associated windows.

If it happens that the first transition chain reaches $T_{\iota_{1}}$, the target torus from the statement of Theorem 1.2, i.e., $\left\{T_{i_{t}}\right\}_{t}$ has no large gaps, the proof is already being completed. If not, we proceed by crossing the first large gap.

4.1.4. Construction of correctly aligned windows across the first large gap. This is the most delicate step of the argument.

In the previous step we have obtained a window $\widetilde{W}_{t_{1}}$ centered at a point $F^{N_{t_{1}}}\left(p_{t_{1}}\right)$ on the torus $T_{i_{t_{1}}}=T_{j_{1}}$, which is the lower bound of the first large gap. Thus, by P3.b, the region between $T_{i_{t_{1}}}$ and $T_{i_{t_{1}+1}}$ that lies ahead is a Birkhoff zone of instability. By Theorem 3.3, there exists a point $p$ close to $T_{i_{t_{1}}}$ which lands near $T_{i_{t_{1}+1}}$ under some iterate $F^{K_{t_{1}}}$. We want to construct windows $\widetilde{W}_{t_{1}}$ about $T_{i_{t_{1}}}$ and $\widehat{W}_{t_{1}+1}$ about $T_{i_{t_{1}+1}}$, such that the former is correctly aligned with the latter under some iterate $F^{K_{t_{1}}}$. We would also want that $\widetilde{W}_{t_{1}}$ is correctly aligned with the windows constructed at the previous step. 


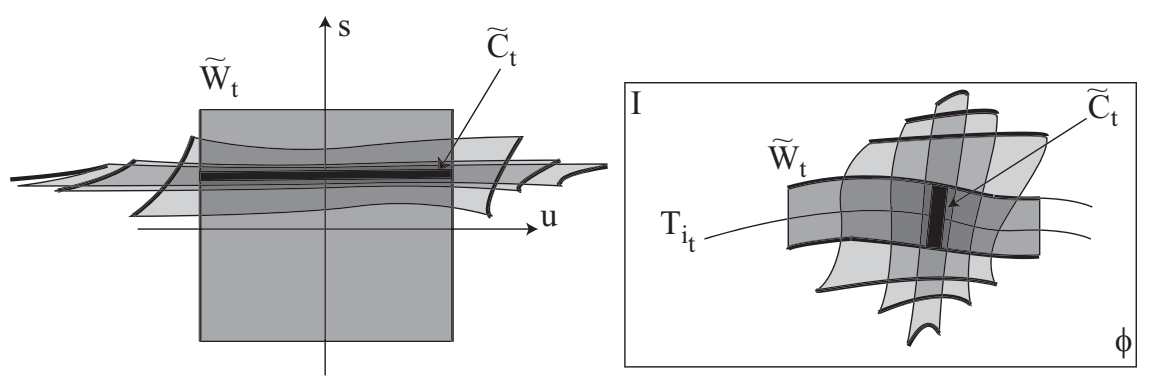

Figure 10. Construction of correctly aligned windows along $T_{i_{t}}$, at the border of a gap - sections along the $(u, s)$-coordinates (left), and sections along the $(\phi, I)$-coordinates (right); the exit sets of the sections are marked by thicker lines.

First, note that due to the correct alignment of the windows $\widetilde{W}_{0}, W_{0}^{\prime}, \ldots, W_{t_{1}}, \widetilde{W}_{t_{1}}$ at the end of Subsection 4.1.4, the intersection

$$
\widetilde{W}_{t_{1}} \cap F^{N_{t_{1}}}\left(W_{t_{1}}\right) \cap F^{N_{t_{1}}}\left(W_{t_{1}-1}^{\prime}\right) \cap \ldots \cap F^{N_{t_{1}}+\ldots+N_{0}+M_{0}}\left(\widehat{W}_{0}\right)
$$

has a component $\widetilde{C}_{t_{1}}$ that is a topological rectangle in $\widetilde{W}_{t_{1}}$. This is not, in general, a rectangle in the local coordinate system $(u, s, \phi, \bar{I})$ near $T_{i_{t_{1}}}$. To $\widetilde{C}_{t_{1}}$ it corresponds in $A$ a topological rectangle, which we denote by $\widetilde{B}_{t_{1}}$, such that

$$
\widetilde{C}_{t_{1}}=\bigcup_{x \in \widetilde{B}_{t_{1}}} \widetilde{\Delta}_{t_{1}}(x),
$$

where $\widetilde{\Delta}_{t_{1}}(x)$ is a rectangle in the $(u, s)$-coordinates, depending on $x \in \widetilde{B}_{t_{1}}$. This topological rectangle $\widetilde{C}_{t_{1}}$ intersects the window $\widetilde{W}_{t_{1}}$ only across its exit set, in a manner that is correctly aligned. See Figure 10. This intersection defines an exit set of $\widetilde{C}_{t_{1}}$, and, implicitly, an exit direction of $\widetilde{B}_{t_{1}}$.

Then note that, by P3.a, there exists a heteroclinic connection at $q_{t_{1}+1}$ between $T_{i_{t_{1}+1}}$ and $T_{i_{t_{1}+2}}$, on the other side of first large gap. We will now construct windows about $T_{i_{1}+1}$, about $q_{t_{1}+1}$, and about $T_{i_{t_{1}+2}}$, with arbitrarily small diameters in the exit directions.

By Subsection 4.1.2, we can choose a pair of windows $\left(W_{t_{1}+1}^{\prime}\right)^{k}$ and $\left(W_{t_{1}+2}\right)^{k}$ at $q_{t_{1}+1}$ that are correctly aligned under the identity map, for $k \geq 0$; we require that the diameters of $\left(W_{t_{1}+1}^{\prime}\right)^{k}$ and $\left(W_{t_{1}+2}\right)^{k}$, measured in the exit directions, approach 0 as $k \rightarrow \infty$. As in Subsection 3.6, we can construct $\left(\widetilde{W}_{t_{1}+2}\right)^{k}$ by $T_{i_{t_{1}+2}}$ such that $\left(W_{t_{1}+2}\right)^{k}$ is correctly aligned with $\widetilde{W}_{t_{1}+2}$ under some iterate $F^{N_{t_{1}+2}}$ that does not depend on $k$; moreover, we can choose $\left(\widetilde{W}_{t_{1}+2}\right)^{k}$ such that its exit set has the center components lying on a pair of tori $T_{\iota_{k}^{\prime}}$ and $T_{\iota_{k}^{\prime \prime}}$ from $\left\{T_{\iota}\right\}_{\iota \in \mathcal{I}}$, such that $\iota_{k}^{\prime} \prec i_{t_{1}+2} \prec \iota_{k}^{\prime \prime}$ and $\iota_{k}^{\prime}, \iota_{k}^{\prime \prime} \rightarrow i_{t_{1}+2}$ as $k \rightarrow \infty$. Also, about $F^{-M_{t_{1}+1}}\left(p_{t_{1}+1}^{\prime}\right) \in T_{t_{1}+1}$ we construct $\left(\widehat{W}_{t_{1}+1}\right)^{k}$ such that is correctly aligned with $\left(W_{t_{1}+1}^{\prime}\right)^{k}$ under $F^{M_{t_{1}+2}}$, where the iterate $F^{-M_{t_{1}+1}}$ is chosen and fixed such that the distance between any point in $F^{-M_{t_{1}+1}}\left(\left(W_{t_{1}+1}^{\prime}\right)^{0}\right)$ and $T_{i_{t_{1}+1}}$, measured with respect to the $(I, u)$ coordinates, is less than $\left(\epsilon_{t_{1}+1} / 2\right)$. The center component of the exit set of $\left(\widehat{W}_{t_{1}+1}\right)^{k}$ consists of a pair of $C^{1}$-curves transverse to $T_{i_{t_{1}+1}}$ in $A$, with the $C^{1}$-distance 


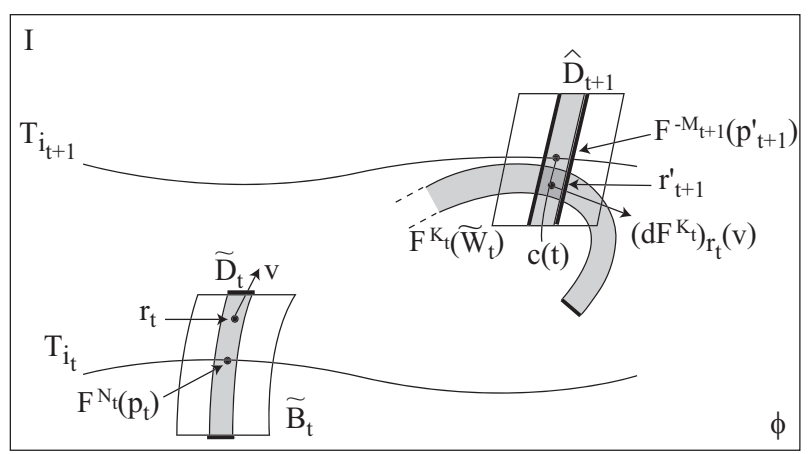

Figure 11. Construction of correctly rectangles that cross the instability zone in a correctly aligned manner.

between the curves approaching 0 as $k \rightarrow \infty$. The limiting position of this pair of curves as $k \rightarrow \infty$ is a $C^{1}$-curve $c(t)$ in $A$ emanating from $F^{-M_{t_{1}+1}}\left(p_{t_{1}+1}^{\prime}\right)$. For each $k$, $\left(\widehat{W}_{t_{1}+1}\right)^{k} \cap A$ is a thin strip in $A$ containing $c(t)$, whose width approaches 0 as $k \rightarrow \infty$. The exit direction of $\left(\widehat{W}_{t_{1}+1}\right)^{k} \cap A$ is transverse to the tangent direction to $c(t)$.

By Lemma 3.4, there exists a point $r_{t_{1}+1}^{\prime}$ on the curve $c(t)$, as close as we wish to $F^{-M_{t_{1}+1}}\left(p_{t_{1}+1}^{\prime}\right)$, and a power $F^{K_{t_{1}}}$ of $F$ such that $r_{t_{1}}=F^{-K_{t_{1}}}\left(r_{t_{1}+1}^{\prime}\right)$ is in $\widetilde{B}_{t_{1}}$. Moreover, we can find a vector $v$ at $r_{t_{1}}$, transverse to the $\phi$-direction, which is mapped by $\left(d F^{K_{t_{1}}}\right)_{r_{t_{1}}}$ into the vector $\left(d F^{K_{t_{1}}}\right)_{r_{t_{1}}}(v)$ at $r_{t_{1}+1}^{\prime}$, that is transverse to the tangent direction to $c$ at $r_{t_{1}+1}^{\prime}$.

Then, inside the topological rectangle $\widetilde{B}_{t_{1}}$, we can construct a thin strip $\widetilde{D}_{t_{1}}$ in $A$ that contains $r_{t_{1}}$, such that the direction of the thin strip at $r_{t_{1}}$ is given by $v$. We also require that the exit set of $\widetilde{D}_{t_{1}}$ is contained in the exit set of $\widetilde{B}_{t_{1}}$. The entry direction of $\widetilde{D}_{t_{1}}$ points in the $\phi$-direction. If we choose the strip $\widetilde{D}_{t_{1}}$ thin enough in the $\phi$-direction, we can ensure that the curve $c(t)$ crosses $F^{K_{t_{1}}}\left(\widetilde{D}_{t_{1}}\right)$ all the way through about the point $r_{t_{1}+1}^{\prime}$, by entering through one entry component and exiting right away through the other entry component. Of course, the curve may enter and exit $F^{K_{t_{1}}}\left(\widetilde{D}_{t_{1}}\right)$ many other times away from the point $r_{t_{1}+1}^{\prime}$. Nevertheless, using Definition 2.2, we can construct a thin strip $\widehat{D}_{t_{1}+1}$ in $A$ around $c(t)$, with exit direction near $r_{t_{1}+1}^{\prime}$ pointing in the direction of $v$, such that $\widetilde{D}_{t_{1}}$ is correctly aligned with $\widehat{D}_{t_{1}+1}$ under $F^{K_{t_{1}}}$. See Figure 11 .

We now choose and fix $k$ sufficiently small such that $\left(\widehat{W}_{t_{1}+1}\right)^{k}$ intersects $A$ in a topological rectangle about $c(t)$ that is contained in the strip $\widehat{D}_{t_{1}+1}$.

This window $\left(\widehat{W}_{t_{1}+1}\right)^{k}$ is, by definition, our new window $\widehat{W}_{t_{1}+1}$ that we will propagate further. To this window it corresponds a pair of windows $W_{t_{1}+1}^{\prime}$ and $W_{t_{1}+2}$ about $q_{t_{1}+1}$ that are correctly aligned under the identity map, and another window $\widetilde{W}_{t_{1}+2}$ about the next torus $T_{t_{1}+2}$. These windows correspond to the previously constructed $\left(W_{t_{1}+1}^{\prime}\right)^{k},\left(W_{t_{1}+2}\right)^{k}$, and $\left(\widetilde{W}_{t_{1}+2}\right)^{k}$, for our chosen $k$. Through our construction, we have ensured that the exit set corresponding to $\widetilde{W}_{t_{1}+2} \cap A$ lies on tori nearby $T_{t_{1}+2}$, from the family $\left\{T_{\iota}\right\}_{\iota \in \mathcal{I}}$. This property is required to carry 


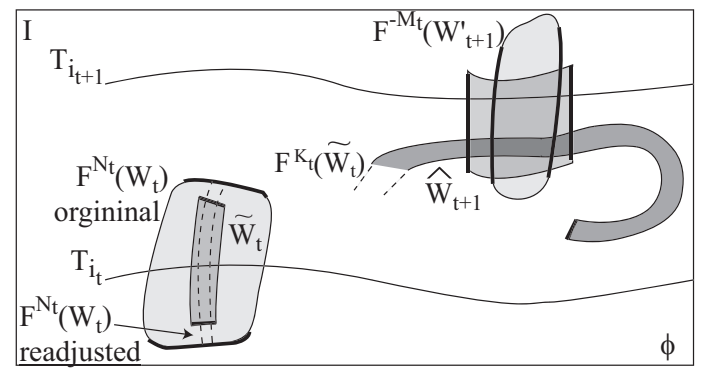

FiguRE 12. Construction of correctly aligned windows across a large gap between $T_{i_{t}}$ and $T_{i_{t+1}}$ - sections along the $(\phi, I)$ coordinates; the exit sets of the sections are marked by thicker lines.

on the construction of correctly aligned windows in a manner similar to Subsection 4.1.3. This will be done explicitly in Subsection 4.2.3.

Now we need to redefine the window $\widetilde{W}_{t_{1}}$. We construct $\widetilde{W}_{t_{1}}$ as a topological rectangle that crosses $\widetilde{C}_{t_{1}}$ in a manner that is correctly aligned, and that intersects $A$ along a 2-dimensional window contained in $\widetilde{B}_{t_{1}}$ and which contains $r_{t_{1}}$. In this way, we can ensure that $\widetilde{W}_{t_{1}} \cap A$ is correctly aligned with $\widehat{W}_{t_{1}+1} \cap A$ under $F^{K_{t_{1}}}$. We adjust the size of the windows $\widetilde{W}_{t_{1}} \cap A$ and $\widehat{W}_{t_{1}+1} \cap A$ in the hyperbolic directions such that $\widetilde{W}_{t_{1}}$ is correctly aligned with $\widehat{W}_{t_{1}+1}$ under $F^{K_{t_{1}}}$. In conclusion we obtain $\widetilde{W}_{t_{1}}$ correctly aligned with $\widehat{W}_{t_{1}+1}$ under $F^{K_{t_{1}}}$.

The fact that we redefined $\widetilde{W}_{t_{1}}$ destroys the previous correct alignments in the sequence $\widetilde{W}_{0}, W_{0}^{\prime}, W_{1}, \widetilde{W}_{1}, \widehat{W}_{1}, \ldots, \widetilde{W}_{t_{1}-1}, \widehat{W}_{t_{1}-1}, W_{t_{1}-1}^{\prime}, W_{t_{1}}, \widetilde{W}_{t_{1}}$ constructed in Subsection 4.1.3. This will require a backwards avalanche of adjustments of the windows constructed along the first transition chain, that will be described in Subsection 4.1.5.

On the other hand, $\widehat{W}_{t_{1}+1} \cap A$ has to be sufficiently thin in the $\phi$-direction in order for $\widetilde{W}_{t_{1}} \cap A$ to be correctly aligned with $\widehat{W}_{t_{1}+1} \cap A$ under $F^{K_{t_{1}}}$. See Figure 12. Consequently, $W_{t_{1}+1}^{\prime}$ has to be sufficiently thin in the exit direction. This will impose forward restrictions, in terms of the smallness in size of the exit directions, for all windows that will be constructed along the second transition chain. Similarly to what we have seen in Subsections 4.1.1 and 4.1.3, if we start with a smallness condition on the size of the exit direction of the window $W_{t_{1}+1}^{\prime}$, this condition will be inherited by all the correctly aligned windows that will be constructed along the next transition chain. However, when the next large gap will be encountered, this will require backwards adjustments on the size of the entry direction. There is no reason at this point why these two types of adjustment can be performed without affecting one another. We will describe a procedure to perform this double adjustment coherently in the induction step in Section 4.2.

4.1.5. Revision of the sequence of correctly aligned windows along the first transition chain. We revise the construction of

$$
\widetilde{W}_{0}, W_{0}^{\prime}, W_{1}, \widetilde{W}_{1}, \widehat{W}_{1}, \ldots, \widetilde{W}_{t_{1}-1}, \widehat{W}_{t_{1}-1}, W_{t_{1}-1}^{\prime}, W_{t_{1}}, \widetilde{W}_{t_{1}}
$$


with $\widetilde{W}_{t_{1}}$ redefined as in the previous section, in order to ensure that each window in the sequence is correctly aligned with the next window under the appropriate power of $F$. By the previous paragraph, the window of $\widetilde{W}_{t_{1}}$ had to be redefined so that it is correctly aligned with $\widetilde{C}_{t_{1}}$, which is a component of the intersection $\widetilde{W}_{t_{1}} \cap F^{N_{t_{1}}}\left(W_{t_{1}}\right) \cap F^{N_{t_{1}}}\left(W_{t_{1}-1}^{\prime}\right) \cap \ldots \cap F^{N_{t_{1}}+\ldots+N_{0}+M_{0}}\left(\widehat{W}_{0}\right)$. Consequently, going backwards in the chain of correctly aligned windows one step at a time, we can select topological rectangles inside all windows constructed up to $\widetilde{W}_{t_{1}}$ such that that each window in the sequence is correctly aligned with the successive window under the appropriate power of $F$. For example, the redefined $W_{t_{1}}$ will be a component of $F^{-N_{t_{1}}}\left(\widetilde{W}_{t_{1}}\right) \cap W_{t_{1}}$, slightly enlarged in the exit direction and slightly diminished in the entry direction in order to ensure correct alignment. Since the redefined windows are just selections of topological rectangles inside the original ones, the number of iterates of $F$ that ensures the correct alignment at each stage is the same as in the original sequence.

At the end of this procedure, we have obtained a new sequence of windows along the first transition chain, for which we use the same notation as before,

$$
\widetilde{W}_{0}, W_{0}^{\prime}, W_{1}, \widetilde{W}_{1}, \widehat{W}_{1}, \ldots, \widetilde{W}_{t_{1}-1}, \widehat{W}_{t_{1}-1}, W_{t_{1}-1}^{\prime}, W_{t_{1}}, \widetilde{W}_{t_{1}},
$$

where the number of iterations $N_{t}, K_{t}, M_{t}$ required to perform correct alignment between successive windows does not change from before. The fact that we do not revise the number of iterates as we revise the construction of windows is quite critical for our argument.

We have also obtained $\widetilde{W}_{t_{1}}$ correctly aligned with $\widehat{W}_{t_{1}+1}$ under $F^{K_{t_{1}}}$. This latter pair of windows crosses the first large gap.

This completes the initial step of the induction process.

4.2. Induction step. We assume that we have already constructed correctly aligned windows along the first $m$ transition chains alternating with the first $m$ large gaps, and we have arrived with the construction at a window $\widehat{W}_{t_{m}+1}$ around the torus $T_{i_{t_{m}+1}}$, which is the upper bound of the $m$-th large gap. As in Subsection 4.1.4, the section $\widehat{W}_{t_{m}+1} \cap A$ of $\widehat{W}_{t_{m}+1}$ contains a point $r_{t_{m}+1}^{\prime}$ which is the image of a point $r_{t_{m}}$ that travels across the Birkhoff zone of instability between $T_{i_{t_{m}}}$ and $T_{i_{t_{m}+1}}$. There is a window $\widetilde{W}_{t_{m}}$ already constructed around $r_{t_{m}}$. The exit direction of $\widetilde{W}_{t_{m}}$ corresponds to the unstable directions and to some direction in $A$ that is transverse to the $\phi$-direction. The exit direction of $\widehat{W}_{t_{m}+1}$ corresponds to the unstable directions and to some direction in $A$ that is transverse to the $\bar{I}$-direction. The section $\widehat{W}_{t_{m}+1} \cap A$ has to be of some prescribed width in the $\bar{I}$-direction (imposed by the correct alignment of $\widehat{W}_{t_{m}+1}$ with $\left.W_{t_{m}+1}^{\prime}\right)$, and sufficiently thin in the $\phi$-direction so that $F^{K_{t_{m}}}\left(\widetilde{W}_{t_{m}}\right) \cap A$ crosses $\widehat{W}_{t_{m}+1} \cap A$ in a manner that is correctly aligned. See Figure 12. We are now going to continue the construction of correctly aligned windows along the next transition chain and across the next large gap. Since most steps of the construction will be similar to the steps in Subsections 4.1.1, 4.1.2, $4.1 .3,4.1 .4$, and 4.1 .5 , we will focus mainly on the differences.

4.2.1. Construction of correctly aligned windows about a heteroclinic point. We construct a pair of windows $W_{t_{m}+1}^{\prime}$ and $W_{t_{m}+2}$ near $q_{t_{m}+1}$ that are correctly aligned under the identity mapping, as described in Subsection 3.6, and such that $\widehat{W}_{t_{m}+1}$ 
is correctly aligned with $W_{t_{m}+1}^{\prime}$ under $F^{m_{t}}$. The windows $W_{t_{m}+1}^{\prime}$ and $W_{t_{m}+2}$ need to be sufficiently small in the exit direction.

4.2.2. Construction of correctly aligned windows by the transversality-torsion mechanism. By the transversality-torsion mechanism, the smallness in the size of the exit direction of $W_{t_{m}+1}^{\prime}$ imposes a restriction on the smallness in the size of the exit direction of $W_{t_{m}+2}$. These smallness condition will propagate forward to the subsequent windows.

4.2.3. Construction of correctly aligned windows along the $(m+1)$-th transition chain. We propagate the construction of correctly aligned windows along the $(m+$ 1)-th transition chain that starts at $T_{i_{t_{m}+1}}$ and ends at $T_{t_{m+1}}$, the lower boundary of the next large gap, and obtain

$$
\widehat{W}_{t_{m}+1}, W_{t_{m}+1}^{\prime}, W_{t_{m}+2}, \widetilde{W}_{t_{m}+2}, \ldots, \widetilde{W}_{t_{m+1}-1}, \widehat{W}_{t_{m+1}-1}, W_{t_{m+1}-1}^{\prime}, W_{i_{t_{m}+1}}, \widetilde{W}_{t_{m+1}},
$$

with $W_{t_{m+1}}$ about the torus $T_{t_{m+1}}$ at the lower boundary of the next large gap, such that any two consecutive windows in the sequence are correctly aligned under identity or under some power $F^{N_{t}}, F^{K_{t}}, F^{M_{t}}$ of $F$. Whenever we want to correctly align two windows $\widehat{W}_{t}$ and $\widetilde{W}_{t}$ about the same torus $T_{t}$, we use the fact that the exit set components of $\widehat{W}_{t} \cap A$ and the entry set components of $\widetilde{W}_{t}$ lie on invariant tori neighboring $T_{t}$ to achieve correct alignment of the center components of the windows under some power $F^{K_{t}}$; see Subsection 4.1.3.

4.2.4. Construction of correctly aligned windows across the $(m+1)$-th large gap. The region between $T_{i_{t_{m+1}}}$ and $T_{i_{t_{m+1}+1}}$ is a Birkhoff zone of instability.

The intersection

$$
\widetilde{W}_{t_{m+1}} \cap F^{N_{t_{m+1}}}\left(W_{t_{m+1}}\right) \cap \ldots \cap F^{N_{t_{m+1}}+\ldots+N_{t_{m+1}}+M_{t_{m+1}}}\left(\widehat{W}_{t_{m+1}}\right),
$$

following all the windows in the preceding transition chain, has a component $\widetilde{C}_{t_{m+1}}$ that is a topological rectangle in $\widetilde{W}_{t_{m+1}}$, of the form

$$
\widetilde{C}_{t_{m+1}}=\bigcup_{x \in \widetilde{B}_{t_{m+1}}} \widetilde{\Delta}_{t_{m+1}}(x),
$$

where $\widetilde{\Delta}_{t_{m+1}}(x)$ is a rectangle in the $(u, s)$-coordinates, and $\widetilde{B}_{t_{m+1}}$ is a rectangle in $A$. The intersection between $\widetilde{C}_{t_{m+1}}$ and the exit set of $\widetilde{W}_{t_{m+1}}$ defines the exit set of $\widetilde{C}_{t_{m+1}}$, and, implicitly, the exit set of $\widetilde{B}_{t_{m+1}}$.

The heteroclinic connection between $T_{i_{t_{m+1}+1}}$ and $T_{i_{t_{m+1}+2}}$ yields a sequence of pairs of windows $\left(W_{t_{m+1}+1}^{\prime}\right)^{k}$ and $\left(W_{t_{m+1}+2}\right)^{k}$, located by the heteroclinic point $q_{t_{m+1}+1}$, that are correctly aligned under the identity, and for which the widths in the exit directions approach 0 as $k \rightarrow \infty$. Then, there exits a curve $c(t)$ in $A$ emerging from the point $F^{-M_{t_{m+1}+2}}\left(p_{t_{m+1}+2}^{\prime}\right)$, and a sequence of windows $\left(\widetilde{W}_{t_{m+1}+1}\right)^{k}$ by $F^{-M_{t_{m+1}+2}}\left(p_{t_{m+1}+2}^{\prime}\right)$, such that $\left(\widetilde{W}_{t_{m+1}+1}\right)^{k} \cap A$ contains the curve $c(t)$ and $\left(\widetilde{W}_{t_{m+1}+1}\right)^{k}$ is correctly aligned with $\left(W_{t_{m+1}+1}^{\prime}\right)^{k}$ for each $k$. By Lemma 3.4 , there exists a point $r_{t_{m+1}} \in \widetilde{B}_{t_{m+1}}$ and a power $F^{K_{t_{m+1}}}$ of $F$ such that $r_{t_{m+1}+1}^{\prime}=F^{K_{t_{m+1}}}\left(r_{t_{m+1}}\right)$ is in $c(t)$. There exists a window $\widetilde{W}_{t_{m+1}}$ about $r_{t_{m+1}}$, with $\widetilde{W}_{t_{m+1}} \cap A \subseteq \widetilde{B}_{t_{m+1}}$, and a window $\left(\widehat{W}_{t_{m+1}+1}\right)^{k}$ about $r_{t_{m+1}+1}^{\prime}$, such that $\widetilde{W}_{t_{m+1}}$ is correctly aligned with $\left(\widehat{W}_{t_{m+1}+1}\right)^{k}$ under $F^{K_{t_{m+1}}}$. In order to have $F^{N_{t_{m+1}}}\left(W_{t_{m+1}}\right)$ 


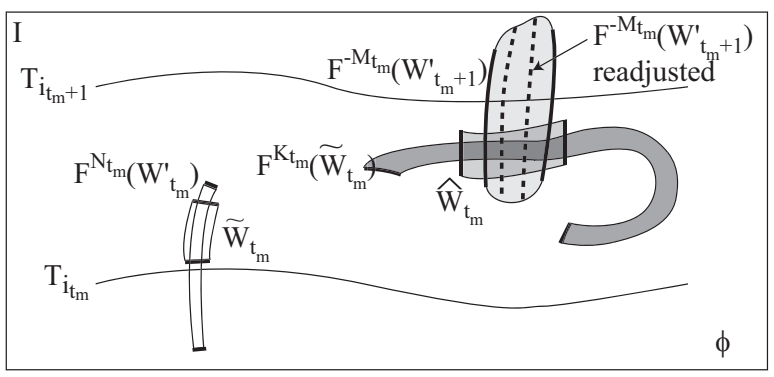

FIGURE 13. Readjustment of the correctly aligned windows across a large gap between $T_{i_{t_{m}}}$ and $T_{i_{t_{m}+1}}$ - sections along the $(\phi, I)$ coordinates; the exit sets of the sections are marked by thicker (continuous or dotted) lines.

crossing $\left(\widetilde{W}_{t_{m+1}}\right)^{k}$ in a manner that is correctly aligned, and $\left(\widehat{W}_{t_{m+1}+1}\right)^{k}$ crossing $F^{-M_{t_{m+1}}}\left(\left(W_{t_{m+1}+1}^{\prime}\right)^{k}\right)$ in a manner that is correctly aligned, we need to choose $k$ sufficiently large so that $\left(\widetilde{W}_{t_{m+1}}\right)^{k} \cap A$ is sufficiently thin in the $\phi$-direction.

4.2.5. Revision of the sequence of correctly aligned windows along the previous transition chain. In the previous step, we have modified the window $\widetilde{W}_{t_{m+1}}$ at the tail of the sequence

$$
W_{t_{m}+1}^{\prime}, W_{t_{m}+2}, \widetilde{W}_{t_{m}+2}, \widehat{W}_{t_{m}+2}, \ldots, \widetilde{W}_{t_{m+1}-1}, \widehat{W}_{t_{1}-1}, W_{t_{m+1}-1}^{\prime}, W_{t_{m+1}}, \widetilde{W}_{t_{m+1}} .
$$

Thus, we need to redefine all the preceding windows in the chain, in order to ensure that each window crosses the successive window in a manner that is correctly aligned, under the appropriate iterate of $F$. The new window of $\widetilde{W}_{t_{m+1}}$ is required to be sufficiently thin in the $\phi$-component of the entry direction. Since $\widetilde{W}_{t_{m+1}}$ was constructed inside the intersection $\widetilde{C}_{t_{m+1}}$, we can propagate it backwards, one step at the time, all the way through, obtaining a new sequence of correctly aligned windows, still denoted by

$$
W_{t_{m}+1}^{\prime}, W_{t_{m}+2}, \widetilde{W}_{t_{m}+2}, \widehat{W}_{t_{m}+2}, \ldots, \widetilde{W}_{t_{m+1}-1}, \widehat{W}_{t_{1}-1}, W_{t_{m+1}-1}^{\prime}, W_{t_{m+1}}, \widetilde{W}_{t_{m+1}} .
$$

The number of iterates it takes to correctly align one window to the next window in the sequence is the same as in the previously constructed sequence, at the beginning of the paragraph.

When this avalanche of backwards adjustments reaches $T_{i_{t_{m}+1}}$, the window $\widehat{W}_{t_{m}+1}$ needs to be made even thinner in the center component of its exit direction. However, making $\widehat{W}_{t_{m}+1} \cap A$ thinner in the $\phi$-direction does not affect at all the crossing of $F^{K_{t_{m}}}\left(\widetilde{W}_{t_{m}}\right) \cap A$. This is a crucial part of the argument: the sequence of the backwards adjustments stops at the upper bound of the previous large gap, and it does not affect the constructions on the previous transition chains and previous gaps. This completes the induction step.

This type of argument, that if a window is correctly aligned with another window, then it is correctly aligned with any sub-window of the latter, was also used in the proof of Theorem 2.5. See Figure 13.

In summary, each crossing of a large gap determines two series of refinements: a backward series of adjustments in the center component of the entry directions 
of the sequence of windows that arrives at the lower bound of the large gap, and a forward series of adjustments in the center component of the exit directions of the sequence of windows that departs from the upper bound of the large gap. Each backward series of adjustments only goes back to the upper bound of the previous large gap, and not any further. One essential ingredient that allows to perform the two kinds of adjustments without harming one another is the existence of 'interior' invariant tori that are limits from above and from below of other transition tori.

\section{FINAL REMARKS}

Remark 5.1. A classical geometric argument for Arnold diffusion is the obstruction argument (see $[1,11,12]$ ). However, the obstruction argument can only be applied for transition chains of tori - primary or secondary - and relies heavily on the Lambda Lemma. In the situation with large gaps that we consider, there are no transition chains that cross the gaps. We are not able to use the standard Lambda Lemma to traverse these large gaps, so the classical obstruction argument does not immediately apply to this situation. It appears possible though ([16, 29]) to adapt the Lambda Lemma in order to use it to cross zones of instability. The method of correctly aligned windows that we employ here can be viewed as an adaptation of the obstruction argument to a situation that combines transition chains and zones of instability. This is quite different from the usual type of application of correctly aligned windows to detect periodic orbits and symbolic dynamics, as, for example, in $[17,18,38,42,43]$; also compare with [30].

Remark 5.2. The method of correctly aligned windows can be implemented numerically to detect orbits with prescribed itineraries, periodic orbits and chaos in concrete system (see, for example [43] and the references listed there). Another technique that is used in proving Arnold diffusion is the scattering map [12]. The scattering map was implemented numerically in the study of the spatial Hill's problem in [13]. It seems possible that the windowing technique can be combined with the scattering map technique for numerical verifications of Arnold diffusion in concrete systems.

Remark 5.3. The result of Birkhoff mentioned in Subsection 3.2 remain valid for positive-tilt maps (see [32]). Consequently, Theorem 1.2 remains true even if the restriction of $F$ to the annulus $A$ is only a positive-tilt map.

\section{ACKNOWLEDGEMENT}

We would like to express our gratitude to Sylvian Crovisier and Patrice le Calvez for their help on constructing an example of a Birkhoff Zone of Instability with $C^{1}$ smooth boundary components. Discussions with Zhihong Xia were helpful in taking their comments and providing the example produced in this paper. We would also like to thank the anonymous referees for their excellent comments and suggestions.

\section{REFERENCES}

[1] V.I. Arnold, Instability of dynamical systems with several degrees of freedom, Sov. Math. Doklady, 5 (1964), 581-585.

[2] S. Aubry, The twist map, the extended Frenkel-Kontorova model and the devil's staircase, Physica D, 7 (1983), 240-258.

[3] G.D. Birkhoff, Surface transformations and their dynamical applications, Acta Math. 43 (1922), 111-229. 
[4] G.D. Birkhoff, Sur quelques courbes fermées remarquables, Bull. Soc. Math. de France 60 (1932), 1-26.

[5] C. Bonatti and S. Crovisier, Récurrence et généricité, Inventiones mathematicae 158 (2004), 33-104.

[6] C.-Q. Cheng and J. Yan, Existence of diffusion orbits in a priori unstable Hamiltonian systems, J. Differential Geom., 67 (2004), 457-517.

[7] C.-Q. Cheng and J. Yan, Arnold diffusion in Hamiltonian systems: 1 a priori unstable case, preprint, 2004.

[8] J. Cresson, Symbolic dynamics and Arnold diffusion, J. Differential Equations, 187 (2003), 269-292.

[9] S. Crovisier, Personal communication.

10] A. Delshams and P. Gutiérrez, Splitting potential and the Poincaré-Melnikov method for whiskered tori in Hamiltonian systems, J. Nonlinear Sci., 10 (2000), 433-476.

[11] A. Delshams, R. de la Llave and T. M. Seara, A geometric approach to the existence of orbits with unbounded energy in generic periodic perturbations by a potential of generic geodesic flows of $\mathbb{T}^{2}$, Comm. Math. Phys., 209 (2000), 353-392.

[12] A. Delshams, R. de la Llave and T. M. Seara, A geometric mechanism for diffusion in hamiltonian systems overcoming the large gap problem: heuristics and rigorous verifications on a model, Memoirs of the American Mathematical Society, 179 (2006), 1-141.

[13] A. Delshams, J.J. Masdemont and P. Roldan, The scattering map in the spatial Hill's problem, preprint.

[14] R. Easton, Orbit Structure near Trajectories Biasymptotic to Invariant Tori, in Classical Mechanics and Dynamical Systems, R. Devaney and Z. Nitecki (eds.), Marcel Dekker, $1981,55-67$.

[15] A. Fathi, Une interprétation plus toplogique de la démonstration du théorème de Birkhoff, appendix of M. Herman, Introduction a l'étude des courbes invariantes par les diffeomorphismes de l'anneau, Asterisque (103-104) 1983.

[16] E. Fontich and P. Martín, Differentiable invariant manifolds for partially hyperbolic tori and a lambda lemma, Nonlinearity, 13 (2000), 1561-1593.

[17] M. Gidea and C. Robinson, Symbolic Dynamics for Transition Tori - II, in New Advances in Celestial Mechanics and Hamiltonian Systems, J. Delgado, E.A. Lacomba, J. Llibre, E. Pérez-Chavela (eds.), Kluwer Academic, 2004, 95-108.

[18] M. Gidea and C. Robinson, Topologically crossing heteroclinic connections to invariant tori, Jour. Diff. Equat., 193 (2003), 49-74.

[19] M. Gidea and R. de la Llave, Topological Methods in the Instability Problem of Hamiltonian Systems, Discrete and Continuous Dynamical Systems - Series A, 14 (2006), 295-328.

[20] M. Gidea and R. de la Llave, Diffusion with optimal time in the large gap problem for Hamiltonian systems, preprint.

[21] P. Golé, Symplectic Twist Maps: Global Variational Techniques, World Scientific, 2001.

[22] A. Haro, Converse KAM theory for monotone positive symplectomorphisms, Nonlinearity 12 (1999), 1299-1322.

[23] M.R. Herman, On the dynamics of Lagrangian tori invariant by symplectic diffeomorphisms, Progress in variational methods in Hamiltonian systems and elliptic equations (L'Aquila, 1990), Pitman Res. Notes Math. Ser., 243, Longman Sci. Tech., Harlow, 1992, 92-112.

[24] M.W. Hirsch, C.C. Pugh and M. Shub, Invariant Manfolds, Lecture Notes in Mathematics 583, Springer-Verlag, 1977.

[25] I. Jungreis, A method for proving that monotone twist maps have no invariant circles, Ergodic Theory. Dynam. Systems 11 (1991), 79-84.

[26] V. Kaloshin, Geometric proofs of Mather's accelerating and connecting theorems, in Topics in Dynamics and Ergodic Theory, S. Bezuglyi and S. Kolyada (eds.), London Mathematical Society, Lecture Notes Series, Cambridge University Press, 2003.

[27] A. Katok and B. Hasselblatt, Introduction to the Modern Theory of Dynamical Systems, Cambridge University Press, Cambridge, 1999.

[28] H. Koch, On the renormalization of Hamiltonian flows and critical invariant tori, Discrete and Continuous Dynamical Systems - Series A, 8 (2002), 633- 646.

[29] R. de la Llave, Personal communication. 
[30] P. Lochack and J-P. Marco, Diffusion times and stability exponents for nearly integrable analytic systems, Central European Journal of Mathematics, 3 (2005), 342-397.

[31] R.S. MacKay and I.C. Percival, Converse KAM: Theory and practice, Commun. Math. Phys. 98 (1985), 469-512.

[32] J. Mather, Non-existence of invariant circles, Ergod. Th. \& Dyn. Sys., 4 (1984), 301-309.

[33] J. Mather, Variational construction of orbits of twist diffeomorphisms, J. Amer. Math. Soc. 4 (1991), 207-263.

[34] J. Mather, Arnold Diffusion. I: Announcement of results, Journal of Mathematical Sciences, 124 (2004), 5275-5289.

[35] R. Moeckel, Generic Drift on Cantor Sets of Annuli, Contemporary Math., 292 (2002), 163-171.

[36] J. Pöschel, Integrability of Hamiltonian systems on Cantor sets, Commun. Pure Appl. Math., 35 (1982), 653-696.

[37] C. Pugh and M. Shub, Linearization of normally hyperbolic diffeomorphisms and flows, Inven. Math., 10 (1970), 187-198.

[38] C. Robinson, Symbolic Dynamics for Transition Tori, Contemporary Math., 292 (2002), 199-208.

[39] A. Stirnemann, Renormalization for golden circles, Comm. Math. Phys. 152 (1993), 369431.

[40] D. Treschev, Multidimensional symplectic separatrix maps, J. Nonlinear Sci., 12 (2002), $27-58$.

[41] D. Treschev, Trajectories in a neighbourhood of asymptotic surfaces of a priori unstable Hamiltonian systems, Nonlinearity, 15(2002), 2033-2052.

[42] P. Zgliczyński, Sharkovskii's Theorem for Multidimensional Perturbations of OneDimensional Maps, Ergod. Th. \& Dyn. Sys., 19 (1999), 1655-1684.

[43] P. Zgliczyński and M. Gidea, Covering relations for multidimensional dynamical systems-I, Journal of Differential Equations, 202 (2004), 32-58.

[44] Z. Xia, Arnold diffusion: a variational construction, in Proceedings of the International Congress of Mathematicians, Vol. II (Berlin, 1998), Doc. Math., Extra Vol. II, $867-877$.

Department of Mathematics, Northeastern Illinois University, Chicago, IL 60625

E-mail address: mgidea@neiu.edu

Department of Mathematics, Northwestern University, Evanston, IL 60208

E-mail address: clark@math.northwestern.edu 